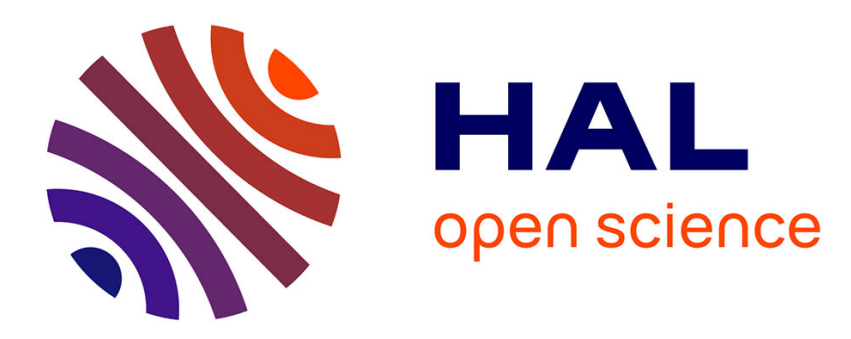

\title{
Selection of weak VARMA models by modified Akaike's information criteria
}

Yacouba Boubacar Mainassara

\section{To cite this version:}

Yacouba Boubacar Mainassara. Selection of weak VARMA models by modified Akaike's information criteria. 2010. hal-00493855v2

\section{HAL Id: hal-00493855 \\ https://hal.science/hal-00493855v2}

Preprint submitted on 13 Sep 2010

HAL is a multi-disciplinary open access archive for the deposit and dissemination of scientific research documents, whether they are published or not. The documents may come from teaching and research institutions in France or abroad, or from public or private research centers.
L'archive ouverte pluridisciplinaire HAL, est destinée au dépôt et à la diffusion de documents scientifiques de niveau recherche, publiés ou non, émanant des établissements d'enseignement et de recherche français ou étrangers, des laboratoires publics ou privés. 


\title{
Selection of weak VARMA models by modified Akaike's information criteria
}

\author{
Y. Boubacar Mainassara ${ }^{a}$ \\ ${ }^{a}$ Université Lille III, EQUIPPE-GREMARS, BP 60 149, 59653 Villeneuve d'Ascq \\ cedex, France.
}

\begin{abstract}
This article considers the problem of order selection of the vector autoregressive moving-average models and of the sub-class of the vector autoregressive models under the assumption that the errors are uncorrelated but not necessarily independent. We propose a modified version of the AIC (Akaike information criterion). This criterion requires the estimation of the matrice involved in the asymptotic variance of the quasi-maximum likelihood estimator of these models. Monte carlo experiments show that the proposed modified criterion estimates the model orders more accurately than the standard AIC and AICc (corrected AIC) in large samples and often in small samples.
\end{abstract}

Key words: AIC, discrepancy, identification, Kullback-Leibler information, model selection, QMLE, order selection, weak VARMA models.

\section{Introduction}

The class of vector autoregressive moving-average (VARMA) models and the sub-class of vector autoregressive (VAR) models are used in time series analysis and econometrics to describe not only the properties of the individual time series but also the possible cross-relationships between the time series (see Reinsel, 1997, Lütkepohl, 2005, 1993).

The parameters estimation is an important step of a $\operatorname{VARMA}(p, q)$ processes modeling. Usually, this estimation is carried out by quasi-maximum likelihood

Email address: mailto:yacouba.boubacarmainassara@univ-lille3.fr (Y. Boubacar Mainassara).

URL: http://perso.univ-lille3.fr/ yboubacarmai/ (Y. Boubacar Mainassara).

Preprint submitted to Elsevier 
or by least squares procedures, given the orders $p$ and $q$ of the model. A companion to the problem of parameter estimation is the problem of model selection, which consists of choosing an appropriate model from a class of candidate models to characterize the data at the hand. The choice of $p$ and $q$ is particularly important because the number of parameters, $(p+q+3) d^{2}$ where $d$ is the number of series, quickly increases with $p$ and $q$, which entails statistical difficulties. If orders lower than the true orders of the $\operatorname{VARMA}(p, q)$ models are selected, the estimate of the parameters will not be consistent and if too high orders are selected, the accuracy of the estimation parameters is likely to be low.

This paper is devoted to the problem of the choice (by minimizing an information criterion) of the VARMA orders under the assumption that the errors are uncorrelated but not necessarily independent. Such models are called weak VARMA, by contrast to the strong VARMA models, that are the standard VARMA usually considered in the time series literature and in which the noise is assumed to be iid. We relax the standard independence assumption to extend the range of application of the VARMA models, allowing us to treat linear representations of general nonlinear processes. The statistical inference of weak ARMA models is mainly limited to the univariate framework (see Francq and Zakoïan, 1998, 2000, 2005, 2007 and Francq, Roy and Zakoïan, 2005).

In the multivariate analysis, important advances have been obtained by Dufour and Pelletier (2005) who study the asymptotic properties of a generalization of the regression-based estimation method proposed by Hannan and Rissanen (1982) under weak assumptions on the innovation process, Francq and Raïssi (2007) who study portmanteau tests for weak VAR models, Boubacar Mainassara and Francq (2009) who study the consistency and the asymptotic normality of the quasi-maximum likelihood estimator (QMLE) for weak VARMA models and Boubacar Mainassara (2009a, 2009b) who studies portmanteau tests for weak VARMA models and studies the estimation of the asymptotic variance of the QMLE of weak VARMA models. Dufour and Pelletier (2005) have proposed a modified information criterion which is a generalization of the information criterion proposed by Hannan and Rissanen (1982).

The choice amongst the models is often made by minimizing an information criterion. The most popular criterion for model selection is the Akaike information criterion (AIC) proposed by Akaike (1973). The AIC was designed to be an approximately unbiased estimator of the expected Kullback-Leibler information of a fitted model. Tsai and Hurvich $(1989,1993)$ derived a bias correction to the AIC for univariate and multivariate autoregressive time series under the assumption that the errors $\epsilon_{t}$ are independent identically distributed (i.e. strong models). The main goal of our paper is to complete the above-mentioned results concerning the statistical analysis of weak VARMA 
models, by proposing a modified version of the AIC criterion.

The paper is organized as follows. Section 2 presents the models that we consider here and summarizes the results on the QMLE asymptotic distribution obtained by Boubacar Mainassara and Francq (2009). In Section 3, we present the $\mathrm{AIC}_{M}$ criterion which we minimize to choose the orders for a weak $\operatorname{VARMA}(p, q)$ models and we establish his overfitting property. This section is also of interest in the univariate framework because, to our knowledge, this model selection criterion has not been studied for weak ARMA models. Numerical experiments are presented in Section 4. The proofs of the main results are collected in the appendix.

\section{$2 \quad$ Model and assumptions}

Consider a $d$-dimensional stationary process $\left(X_{t}\right)$ satisfying a structural $\operatorname{VARMA}\left(p_{0}, q_{0}\right)$ representation of the form

$$
A_{00} X_{t}-\sum_{i=1}^{p_{0}} A_{0 i} X_{t-i}=B_{00} \epsilon_{t}-\sum_{i=1}^{q_{0}} B_{0 i} \epsilon_{t-i}, \quad \forall t \in \mathbb{Z}=\{0, \pm 1, \ldots\}
$$

where $\epsilon_{t}$ is a white noise, namely a stationary sequence of centered and uncorrelated random variables with a non singular variance $\Sigma_{0}$. The structural forms are mainly used in econometrics to introduce instantaneous relationships between economic variables. Of course, constraints are necessary for the identifiability of these representations. Let $\left[A_{00} \ldots A_{0 p_{0}} B_{00} \ldots B_{0 q_{0}} \Sigma_{0}\right]$ be the $d \times\left(p_{0}+q_{0}+3\right) d$ matrix of all the coefficients, without any constraint. The parameter of interest is denoted $\theta_{0}$, where $\theta_{0}$ belongs to the parameter space $\Theta_{p_{0}, q_{0}} \subset \mathbb{R}^{k_{0}}$, and $k_{0}$ is the number of unknown parameters, which is typically much smaller that $\left(p_{0}+q_{0}+3\right) d^{2}$. The matrices $A_{00}, \ldots A_{0 p_{0}}, B_{00}, \ldots B_{0 q_{0}}$ involved in (1) and $\Sigma_{0}$ are specified by $\theta_{0}$. More precisely, we write $A_{0 i}=A_{i}\left(\theta_{0}\right)$ and $B_{0 j}=B_{j}\left(\theta_{0}\right)$ for $i=0, \ldots, p_{0}$ and $j=0, \ldots, q_{0}$, and $\Sigma_{0}=\Sigma\left(\theta_{0}\right)$. We need the following assumptions used by Boubacar Mainassara and Francq (2009), hereafter BMF, to ensure the consistence and the asymptotic normality of the QMLE.

A1: The functions $\theta \mapsto A_{i}(\theta) i=0, \ldots, p, \theta \mapsto B_{j}(\theta) j=0, \ldots, q$ and $\theta \mapsto \Sigma(\theta)$ admit continuous third order derivatives for all $\theta \in \Theta_{p, q}$.

For simplicity we now write $A_{i}, B_{j}$ and $\Sigma$ instead of $A_{i}(\theta), B_{j}(\theta)$ and $\Sigma(\theta)$. Let $A_{\theta}(z)=A_{0}-\sum_{i=1}^{p} A_{i} z^{i}$ and $B_{\theta}(z)=B_{0}-\sum_{i=1}^{q} B_{i} z^{i}$.

A2: For all $\theta \in \Theta_{p, q}$, we have $\operatorname{det} A_{\theta}(z) \operatorname{det} B_{\theta}(z) \neq 0$ for all $|z| \leq 1$; A3: We have $\theta_{0} \in \Theta_{p_{0}, q_{0}}$, where $\Theta_{p_{0}, q_{0}}$ is compact; A4: The process $\left(\epsilon_{t}\right)$ 
is stationary and ergodic; A5: For all $\theta \in \Theta_{p, q}$ such that $\theta \neq \theta_{0}$, either the transfer functions $A_{0}^{-1} B_{0} B_{\theta}^{-1}(z) A_{\theta}(z) \neq A_{00}^{-1} B_{00} B_{\theta_{0}}^{-1}(z) A_{\theta_{0}}(z)$ for some $z \in \mathbb{C}$, or $A_{0}^{-1} B_{0} \Sigma B_{0}^{\prime} A_{0}^{-1^{\prime}} \neq A_{00}^{-1} B_{00} \Sigma_{0} B_{00}^{\prime} A_{00}^{-1^{\prime}} ;$ A6: We have $\theta_{0} \in \stackrel{\circ}{\Theta}_{p_{0}, q_{0}}$, where $\stackrel{\circ}{\Theta}_{p_{0}, q_{0}}$ denotes the interior of $\Theta_{p_{0}, q_{0}}$; A7: We have $E\left\|\epsilon_{t}\right\|^{4+2 \nu}<\infty$ and $\sum_{k=0}^{\infty}\left\{\alpha_{\epsilon}(k)\right\}^{\frac{\nu}{2+\nu}}<\infty$ for some $\nu>0$.

The reader is referred to BMF for a discussion of these assumptions. Note that $\left(\epsilon_{t}\right)$ can be replaced by $\left(X_{t}\right)$ in $\mathbf{A} \mathbf{4}$, because $X_{t}=A_{\theta_{0}}^{-1}(L) B_{\theta_{0}}(L) \epsilon_{t}$ and $\epsilon_{t}=B_{\theta_{0}}^{-1}(L) A_{\theta_{0}}(L) X_{t}$, where $L$ stands for the backward operator. Note that from $\mathbf{A} \mathbf{1}$ the matrices $A_{0}$ and $B_{0}$ are invertible. Introducing the innovation process $e_{t}=A_{00}^{-1} B_{00} \epsilon_{t}$, the structural representation $A_{\theta_{0}}(L) X_{t}=B_{\theta_{0}}(L) \epsilon_{t}$ can be rewritten as the reduced VARMA representation

$$
X_{t}-\sum_{i=1}^{p} A_{00}^{-1} A_{0 i} X_{t-i}=e_{t}-\sum_{i=1}^{q} A_{00}^{-1} B_{0 i} B_{00}^{-1} A_{00} e_{t-i}
$$

We thus recursively define $\tilde{e}_{t}(\theta)$ for $t=1, \ldots, n$ by

$$
\tilde{e}_{t}(\theta)=X_{t}-\sum_{i=1}^{p} A_{0}^{-1} A_{i} X_{t-i}+\sum_{i=1}^{q} A_{0}^{-1} B_{i} B_{0}^{-1} A_{0} \tilde{e}_{t-i}(\theta),
$$

with initial values $\tilde{e}_{0}(\theta)=\cdots=\tilde{e}_{1-q}(\theta)=X_{0}=\cdots=X_{1-p}=0$. The gaussian quasi-likelihood is given by

$$
\tilde{\mathrm{L}}_{n}(\theta)=\prod_{t=1}^{n} \frac{1}{(2 \pi)^{d / 2} \sqrt{\operatorname{det} \Sigma_{e}}} \exp \left\{-\frac{1}{2} \tilde{e}_{t}^{\prime}(\theta) \Sigma_{e}^{-1} \tilde{e}_{t}(\theta)\right\}, \Sigma_{e}=A_{0}^{-1} B_{0} \Sigma B_{0}^{\prime} A_{0}^{-1^{\prime}} .
$$

A quasi-maximum likelihood estimator of $\theta$ is a measurable solution $\hat{\theta}_{n}$ of

$$
\hat{\theta}_{n}=\arg \max _{\theta \in \Theta} \tilde{\mathrm{L}}_{n}(\theta) .
$$

We now use the matrix $M_{\theta_{0}}$ of the coefficients of the reduced form to that made by BMF, where

$$
M_{\theta_{0}}=\left[A_{00}^{-1} A_{01}: \cdots: A_{00}^{-1} A_{0 p}: A_{00}^{-1} B_{01} B_{00}^{-1} A_{00}: \cdots: A_{00}^{-1} B_{0 q} B_{00}^{-1} A_{00}: \Sigma_{e 0}\right] .
$$

We denote by $\operatorname{vec}(A)$ the vector obtained by stacking the columns of $A$. Now we need an assumption which specifies how this matrix depends on the parameter $\theta_{0}$. Let $\dot{M}_{\theta_{0}}$ be the matrix $\partial \operatorname{vec}\left(M_{\theta}\right) / \partial \theta^{\prime}$ evaluated at $\theta_{0}$.

A8: The matrix $\dot{M}_{\theta_{0}}$ is of full rank $k_{0}$.

Under Assumptions A1-A8, BMF showed the consistency $\left(\hat{\theta}_{n} \rightarrow \theta_{0}\right.$ a.s as $n \rightarrow \infty)$ and the asymptotic normality of the QMLE:

$$
\sqrt{n}\left(\hat{\theta}_{n}-\theta_{0}\right) \stackrel{\mathcal{L}}{\rightarrow} \mathcal{N}\left(0, \Omega:=J^{-1} I J^{-1}\right),
$$


where $J=J\left(\theta_{0}\right)$ and $I=I\left(\theta_{0}\right)$, with

$$
J(\theta)=\lim _{n \rightarrow \infty} \frac{2}{n} \frac{\partial^{2}}{\partial \theta \partial \theta^{\prime}} \log \tilde{\mathrm{L}}_{n}(\theta) \quad \text { a.s. } \quad \text { and } \quad I(\theta)=\lim _{n \rightarrow \infty} \operatorname{Var} \frac{2}{\sqrt{n}} \frac{\partial}{\partial \theta} \log \tilde{\mathrm{L}}_{n}(\theta) .
$$

Note that, for VARMA models in reduced form, it is not very restrictive to assume that the coefficients $A_{0}, \ldots, A_{p}, B_{0}, \ldots, B_{q}$ are functionally independent of the coefficient $\Sigma_{e}$. Thus we can write $\theta=\left(\theta^{(1)^{\prime}}, \theta^{(2)^{\prime}}\right)^{\prime}$, where $\theta^{(1)} \in \mathbb{R}^{k_{1}}$ depends on $A_{0}, \ldots, A_{p}$ and $B_{0}, \ldots, B_{q}$, and where $\theta^{(2)} \in \mathbb{R}^{k_{2}}$ depends on $\Sigma_{e}$, with $k_{1}+k_{2}=k_{0}$. With some abuse of notation, we will then write $e_{t}(\theta)=e_{t}\left(\theta^{(1)}\right)$.

A9: With the previous notation $\theta=\left(\theta^{(1)^{\prime}}, \theta^{(2)^{\prime}}\right)^{\prime}$, where $\theta^{(2)}=D$ vec $\Sigma_{e}$ for some matrix $D$ of size $k_{2} \times d^{2}$.

\section{Identification of VARMA models}

Let $\tilde{\ell}_{n}(\theta)=-2 n^{-1} \log \tilde{\mathrm{L}}_{n}(\theta)$ and $e_{t}(\theta)=A_{0}^{-1} B_{0} B_{\theta}^{-1}(L) A_{\theta}(L) X_{t}$. In BMF, it is shown that $\ell_{n}(\theta)=\tilde{\ell}_{n}(\theta)+o(1)$ a.s, where

$$
\ell_{n}(\theta):=-\frac{2}{n} \log \mathrm{L}_{n}(\theta)=\frac{1}{n} \sum_{t=1}^{n}\left\{d \log (2 \pi)+\log \operatorname{det} \Sigma_{e}+e_{t}^{\prime}(\theta) \Sigma_{e}^{-1} e_{t}(\theta)\right\}
$$

It is also shown uniformly in $\theta \in \Theta_{p, q}$ that

$$
\frac{\partial \ell_{n}(\theta)}{\partial \theta}=\frac{\partial \tilde{\ell}_{n}(\theta)}{\partial \theta}+o(1) \quad \text { a.s. }
$$

The same equality holds for the second-order derivatives of $\tilde{\ell}_{n}$.

Note that, minimizing the Kullback-Leibler information of any approximating (or candidate) model, characterized by the parameter vector $\theta$, is equivalent to minimizing the contrast (or the discrepancy between the approximating and the true models) defined by $\Delta(\theta):=E\left\{-2 \log \mathrm{L}_{n}(\theta)\right\}$. Omitting the constant $n d \log (2 \pi)$, we find that

$$
\Delta(\theta)=n \log \operatorname{det} \Sigma_{e}+n \operatorname{Tr}\left(\Sigma_{e}^{-1} S(\theta)\right)
$$

where $S(\theta)=E e_{1}(\theta) e_{1}^{\prime}(\theta)$. The following Lemma shows that the application $\theta \mapsto \Delta(\theta)$ is minimal for $\theta=\theta_{0}$.

Lemma 1 For all $\theta \in \bigcup_{p, q \in \mathbb{N}} \Theta_{p, q}$, we have $\Delta(\theta) \geq \Delta\left(\theta_{0}\right)$. 
Let $X=\left(X_{1}, \ldots, X_{n}\right)$ be observation of a process satisfying the VARMA representation (1). Let, $\hat{e}_{t}=\tilde{e}_{t}\left(\hat{\theta}_{n}\right)$ be the QMLE residuals of a candidate VARMA model when $p>0$ or $q>0$, and let $\hat{e}_{t}=e_{t}=X_{t}$ when $p=q=0$. When $p+q \neq 0$, we have $\hat{e}_{t}=0$ for $t \leq 0$ and $t>n$, and

$$
\hat{e}_{t}=X_{t}-\sum_{i=1}^{p} A_{0}^{-1}\left(\hat{\theta}_{n}\right) A_{i}\left(\hat{\theta}_{n}\right) \hat{X}_{t-i}+\sum_{i=1}^{q} A_{0}^{-1}\left(\hat{\theta}_{n}\right) B_{i}\left(\hat{\theta}_{n}\right) B_{0}^{-1}\left(\hat{\theta}_{n}\right) A_{0}\left(\hat{\theta}_{n}\right) \hat{e}_{t-i}
$$

for $t=1, \ldots, n$, with $\hat{X}_{t}=0$ for $t \leq 0$ and $\hat{X}_{t}=X_{t}$ for $t \geq 1$.

In view of Lemma 1 , it is natural to minimize an estimation of the theoretical criterion $E \Delta\left(\hat{\theta}_{n}\right)$. Of course, $E \Delta\left(\hat{\theta}_{n}\right)$ is unknown, but it can be estimated if certain additional assumptions are made. Note that $E \Delta\left(\hat{\theta}_{n}\right)$ can be interpreted as the average discrepancy when one uses the model of parameter $\hat{\theta}_{n}$.

\subsection{Estimating the discrepancy}

Let $J_{11}$ and $I_{11}$ be respectively the upper-left block of the matrices $J$ and $I$, with appropriate size. The AIC was designed to provide an approximately unbiased estimator of $E \Delta\left(\hat{\theta}_{n}\right)$. In this Section, we will adapt to weak VARMA models the corrected AIC version (AICc) developed by Tsai and Hurvich (1989, 1993) for the univariate and the multivariate strong autoregressive models. Under Assumptions A1-A9, an approximately unbiased estimator of $E \Delta\left(\hat{\theta}_{n}\right)$ is given by

$$
\mathrm{AIC}_{M}:=n \log \operatorname{det} \hat{\Sigma}_{e}+\frac{n^{2} d^{2}}{n d-k_{1}}+\frac{n d}{2\left(n d-k_{1}\right)} \operatorname{Tr}\left(\hat{I}_{11, n} \hat{J}_{11, n}^{-1}\right)
$$

where $\hat{J}_{11, n}$ and $\hat{I}_{11, n}$ are respectively consistent estimators of the matrice $J_{11}$ and $I_{11}$ (see Section 4 of BMF).

Remark 1 Given a collection of competing families of approximating models, the one that minimizes $E \Delta\left(\hat{\theta}_{n}\right)$ might be preferred. For model selection, we then choose $\hat{p}$ and $\hat{q}$ as the set which minimizes the information criterion (3).

Remark 2 In the strong VARMA case, i.e. when $\mathbf{A} 4$ is replaced by the assumption that $\left(\epsilon_{t}\right)$ is iid, we have $I_{11}=2 J_{11}$, so that $\operatorname{Tr}\left(I_{11} J_{11}^{-1}\right)=2 k_{1}$. In this case, the $\mathrm{AIC}_{M}$ takes the following form

$$
\mathrm{AIC}_{M}^{*}:=n \log \operatorname{det} \hat{\Sigma}_{e}+n d+\frac{n d}{n d-k_{1}} 2 k_{1}=\mathrm{AICc}
$$




\subsection{Other decomposition of the discrepancy}

In Section 3.1, the minimal discrepancy (contrast) has been approximated by $-2 E \log \mathrm{L}_{n}\left(\hat{\theta}_{n}\right)$ (the expectation is taken under the true model $X$ ). Note that studying this average discrepancy is too difficult because of the dependance between $\hat{\theta}_{n}$ and $X$. An alternative slightly different but equivalent interpretation for arriving at the expected discrepancy quantity $E \Delta\left(\hat{\theta}_{n}\right)$, as a criterion for judging the quality of an approximating model, is obtained by supposing $\hat{\theta}_{n}$ be the QMLE of $\theta$ based on the observation $X$ and let $Y=\left(Y_{1}, \ldots, Y_{n}\right)$ be independent observation of a process satisfying the VARMA representation (1) (i.e. $X$ and $Y$ independent observations satisfying the same process). Then, we may be interested in approximating the distribution of $\left(Y_{t}\right)$ by using $\mathrm{L}_{n}\left(Y, \hat{\theta}_{n}\right)$. So we consider the discrepancy for the approximating model (model $Y$ ) that uses $\hat{\theta}_{n}$ and, thus, it is generally easier to search a model that minimizes

$$
C\left(\hat{\theta}_{n}\right):=-2 E_{Y} \log \mathrm{L}_{n}\left(\hat{\theta}_{n}\right),
$$

where $E_{Y}$ denotes the expectation under the candidate model $Y$. Since $\hat{\theta}_{n}$ and $Y$ are independent, $C\left(\hat{\theta}_{n}\right)$ is the same quantity as the expected discrepancy $E \Delta\left(\hat{\theta}_{n}\right)$. A model minimizing (4) can be interpreted as a model that will do globally the best job on an independent copy of $X$, but this model may not be the best for the data at hand. The average discrepancy can be decomposed into

$$
C\left(\hat{\theta}_{n}\right)=-2 E_{X} \log \mathrm{L}_{n}\left(\hat{\theta}_{n}\right)+a_{1}+a_{2},
$$

where $a_{1}=-2 E_{X} \log \mathrm{L}_{n}\left(\theta_{0}\right)+2 E_{X} \log \mathrm{L}_{n}\left(\hat{\theta}_{n}\right)$ and $a_{2}=-2 E_{Y} \log \mathrm{L}_{n}\left(\hat{\theta}_{n}\right)+$ $2 E_{X} \log \mathrm{L}_{n}\left(\theta_{0}\right)$. The QMLE satisfies $\log \mathrm{L}_{n}\left(\hat{\theta}_{n}\right) \geq \log \mathrm{L}_{n}\left(\theta_{0}\right)$ almost surely, thus $a_{1}$ can be interpreted as the average over-adjustment (over-fitting) of this QMLE. Now, note that $E_{X} \log \mathrm{L}_{n}\left(\theta_{0}\right)=E_{Y} \log \mathrm{L}_{n}\left(\theta_{0}\right)$, thus $a_{2}$ can be interpreted as an average cost due to the use of the estimated parameter instead of the optimal parameter, when the model is applied to an independent replication of $X$. We now discuss the regularity conditions needed for $a_{1}$ and $a_{2}$ to be equivalent, in the following Proposition.

Proposition 1 Under Assumptions A1-A9, $a_{1}$ and $a_{2}$ are both equivalent to $2^{-1} \operatorname{Tr}\left(I_{11} J_{11}^{-1}\right)$, as $n \rightarrow \infty$.

In view of Proposition 1, in the weak VARMA case, the AIC formula denoted

$$
\mathrm{AIC}_{W}:=-2 \log \mathrm{L}_{n}\left(\hat{\theta}_{n}\right)+\operatorname{Tr}\left(\hat{I}_{11} \hat{J}_{11}^{-1}\right)
$$

is an approximately unbiased estimate of the contrast $C\left(\hat{\theta}_{n}\right)$. Model selection is then obtained by minimizing (5) over the candidate models.

Remark 3 In the strong VARMA case, we have $\operatorname{Tr}\left(I_{11} J_{11}^{-1}\right)=2 k_{1}$. There- 
fore, $a_{1}$ and $a_{2}$ are both equivalent to $k_{1}=\operatorname{dim}\left(\theta_{0}^{(1)}\right)$ (we retrieve the result obtained by Findley, 1993). In this case, the $\mathrm{AIC}_{W}$ formula takes the more conventional form

$$
\mathrm{AIC}=-2 \log \mathrm{L}_{n}\left(\hat{\theta}_{n}\right)+2 k_{1}
$$

\subsection{Overfitting property of the $A I C_{M}$ criterion}

For any models with $k$-dimensional parameter, the $\mathrm{AIC}_{M}$ criterion given in (3) can be rewritten as

$$
\mathrm{AIC}_{M}(k)=n \log \operatorname{det} \hat{\Sigma}_{e}(k)+\frac{n^{2} d^{2}}{n d-k}+\frac{n d}{2(n d-k)} c_{k}
$$

where $c_{k}=\operatorname{Tr}\left(I_{11}\left(\hat{\theta}_{n, k}\right) J_{11}^{-1}\left(\hat{\theta}_{n, k}\right)\right)$ and $\hat{\Sigma}_{e}(k)=\Sigma_{e}\left(\hat{\theta}_{n, k}\right)$.

We define an overfitted model as a model that has more parameters than the true model. Overfitting is analysed here by comparing the model of true orders $p_{0}$ and $q_{0}$ and an overfitted model of orders $p^{\prime}=p_{0}+\ell_{1}$ and $q^{\prime}=q_{0}+\ell_{2}$, where the integers $\ell_{1}, \ell_{2}>0$. Recall that, for the true VARMA model in the reduced form, the number of unknown parameters in VAR and MA parts is $k_{1}=d^{2}\left(p_{0}+q_{0}\right)$. By analog, let $k_{1}^{\prime}=d^{2}\left(p^{\prime}+q^{\prime}\right)$ the number of parameters without any constraints of the overfitted model. Note that, $k_{1}^{\prime}=k_{1}+\ell$ where $\ell=d^{2}\left(\ell_{1}+\ell_{2}\right)$ and let $c_{\ell}=c_{k_{1}^{\prime}}-c_{k_{1}}$. The overfitting property of the $\mathrm{AIC}_{M}$ criterion is described here through the probability of overfitting. The following Lemma gives the overfitting property of the VARMA models.

Proposition 2 The $A I C_{M}$ criterion overfits if $A I C_{M}\left(k_{1}^{\prime}\right)<A I C_{M}\left(k_{1}\right)$. The modified probability that the $A I C_{M}$ criterion selects the overfitted model is

$$
\mathbf{P}_{W}:=P\left\{A I C_{M}\left(k_{1}+\ell\right)<A I C_{M}\left(k_{1}\right)\right\}=P\left\{\chi_{\ell}^{2}>\frac{2 \ell+c_{\ell}}{2}\right\}
$$

Remark 4 In the strong VARMA case, i.e. when A4 is replaced by the assumption that $\left(\epsilon_{t}\right)$ is iid, we have $c_{\ell}=2 \ell$. In this case, the probability that the $\mathrm{AIC}_{M}$ criterion selects the overfitted model takes the following form

$$
\mathbf{P}_{S}:=P\left\{\operatorname{AIC}_{M}\left(k_{1}+\ell\right)<\operatorname{AIC}_{M}\left(k_{1}\right)\right\}=P\left\{\chi_{\ell}^{2}>2 \ell\right\}
$$

From Table 1, it is clear that the $\mathrm{AIC}_{M}$ criterion is not consistent in the strong VAR case, since his probability of overfitting is not zero. 
Table 1

The calculated values for the standard version of asymptotic probabilities of overfitting by $\ell=d^{2} \ell_{1}$ parameters for strong bivariate VAR model.

\begin{tabular}{lrrrrr}
\hline \hline$\ell_{1}$ & 1 & 2 & 3 & 4 & 5 \\
\hline $\mathbf{P}_{S}$ & 0.0915782 & 0.04238011 & 0.02034103 & 0.00999978 & 0.004995412 \\
\hline$\ell_{1}$ & 6 & 7 & 8 & 9 & 10 \\
\hline $\mathbf{P}_{S}$ & 0.002524130 & 0.001286361 & 0.0006599276 & 0.0003403570 & 0.0001763029 \\
\hline \hline
\end{tabular}

\section{Numerical illustrations}

In this section, by means of Monte Carlo experiments, we present the results of simulations study on small and large sample performance of several AIC criteria introduced in this paper. The numerical illustrations of this section are made with the software $\mathrm{R}$ (see http://cran.r-project.org/). We generate VAR models, with several choices of their innovation process $\left(\epsilon_{t}\right)$. Firstly, we consider the strong case in which $\left(\epsilon_{t}\right)$ is defined by

$$
\left(\begin{array}{c}
\epsilon_{1, t} \\
\epsilon_{2, t}
\end{array}\right) \sim \operatorname{IID} \mathcal{N}\left(0, I_{2}\right)
$$

The same experiment is repeated for three weak choices for $\left(\epsilon_{t}\right)$. In the first one, we assume that $\left(\epsilon_{t}\right)$ is an $\operatorname{ARCH}(1)$ model:

$$
\left(\begin{array}{c}
\epsilon_{1, t} \\
\epsilon_{2, t}
\end{array}\right)=\left(\begin{array}{cc}
h_{11, t} & 0 \\
0 & h_{22, t}
\end{array}\right)\left(\begin{array}{l}
\eta_{1, t} \\
\eta_{2, t}
\end{array}\right), \quad \text { with }\left(\begin{array}{c}
\eta_{1, t} \\
\eta_{2, t}
\end{array}\right) \sim \operatorname{IID} \mathcal{N}\left(0, I_{2}\right)
$$

and where

$$
\left(\begin{array}{l}
h_{11, t}^{2} \\
h_{22, t}^{2}
\end{array}\right)=\left(\begin{array}{l}
0.3 \\
0.2
\end{array}\right)+\left(\begin{array}{cc}
0.45 & 0 \\
0.4 & 0.25
\end{array}\right)\left(\begin{array}{c}
\epsilon_{1, t-1}^{2} \\
\epsilon_{2, t-1}^{2}
\end{array}\right)
$$

In two other sets of experiments, we assume that $\left(\epsilon_{t}\right)$ is defined by

$$
\left(\begin{array}{c}
\epsilon_{1, t} \\
\epsilon_{2, t}
\end{array}\right)=\left(\begin{array}{c}
\eta_{1, t} \eta_{2, t-1} \eta_{1, t-2} \\
\eta_{2, t} \eta_{1, t-1} \eta_{2, t-2}
\end{array}\right), \quad \text { with }\left(\begin{array}{c}
\eta_{1, t} \\
\eta_{2, t}
\end{array}\right) \sim \operatorname{IID} \mathcal{N}\left(0, I_{2}\right)
$$


and then by

$$
\left(\begin{array}{c}
\epsilon_{1, t} \\
\epsilon_{2, t}
\end{array}\right)=\left(\begin{array}{c}
\eta_{1, t}\left(\left|\eta_{1, t-1}\right|+1\right)^{-1} \\
\eta_{2, t}\left(\left|\eta_{2, t-1}\right|+1\right)^{-1}
\end{array}\right), \quad \text { with }\left(\begin{array}{c}
\eta_{1, t} \\
\eta_{2, t}
\end{array}\right) \sim \operatorname{IID} \mathcal{N}\left(0, I_{2}\right),
$$

These noises are direct extensions of those defined by Romano and Thombs (1996) in the univariate case.

We used the spectral estimator $\hat{I}^{\mathrm{SP}}:=\hat{\boldsymbol{\Phi}}_{r}^{-1}(1) \hat{\Sigma}_{\hat{u}_{r}} \hat{\boldsymbol{\Phi}}_{r}^{\prime-1}(1)$ of the matrix $I$ defined in Theorem 3 of BMF. In this theorem, the AR order $r=r(n)$ is automatically selected by BIC criterion in the weak models (in this case, Theorem 3 requires that $r \rightarrow \infty$ ), using the function VARselect () of the vars $R$ package. In the strong case we can be shown that, the AR spectral estimator is consistent with any fixed value of $r$ (or $r=o\left(n^{1 / 3}\right)$ as in Theorem 3 and we took $r=1$. The matrix $J$ can easily be estimated by its empirical counterpart. The reader is referred to Section 4 in BMF for a discussion of these estimators involved in our modified criterion.

The corresponding relative rejection frequencies to the orders chosen are displayed in bold type in Tables 2,3 and 4 .

We simulated $N$ independent trajectories of different sizes of a bivariate VAR(1) model with the strong Gaussian and weak noise above-mentioned. We took $N=1,000$ when the sample size $n \leq 2000$ and $N=1,00$ in the opposite case. For each of these $N$ replications, we will fit 6 bivariate candidates models (i.e. $\operatorname{VAR}(k)$ models with $k=1, \ldots, 6)$. The quasi-maximum likelihood (QML) method was used to fit VAR models of order $1, \ldots, 6$. The standard and modified versions of AIC criteria were used to select among the candidate models. To generate the strong and weak VAR(1) models, we consider the bivariate model of the form:

$$
\left(\begin{array}{l}
X_{1 t} \\
X_{2 t}
\end{array}\right)=\left(\begin{array}{ll}
0.5 & 0.1 \\
0.4 & 0.5
\end{array}\right)\left(\begin{array}{l}
X_{1 t-1} \\
X_{2 t-1}
\end{array}\right)+\left(\begin{array}{l}
\epsilon_{1 t} \\
\epsilon_{2 t}
\end{array}\right), \quad \Sigma_{0}=\left[\begin{array}{ll}
1 & 0 \\
0 & 1
\end{array}\right]
$$

Table 2 displays the relative frequency (in \%) of the order selected by various standard and modified versions of the AIC criteria of a strong (Model I) candidates models, over the $N=1,000$ independent replications. In view of the observed relative frequency, the order $p=1$ (i.e. $\operatorname{VAR}(1)$ model) is selected by all versions of the AIC criteria and they have the similar performance.

Table 3 displays the relative frequency (in \%) of the order selected by various standard and modified versions of the AIC criteria of a strong (Model I) and weak (Model II, with error term (8)) candidates models, over the $N$ indepen- 
dent replications. Table 3 shows that the standard AIC criteria clearly did not perform well here when $n \geq 500$, and they have tendency to overestimate the order $p$. When $n=500$ the order $p=1$ is selected by all versions of the AIC criteria, but the modified criterion has better performed. As expected, when $n \geq 2000$ the standard AIC criteria select a weak $\operatorname{VAR}(2)$ model. By contrast, a VAR(1) model is selected by a modified criterion for all values of $n$ and its performance is increasing with $n$.

Table 4 displays the relative frequency (in \%) of the order selected by various standard and modified versions of the AIC criteria of a weak $\operatorname{VAR}(k)$ candidates models for $k=1, \ldots, 6$, firstly with error term (7) (Model III) and secondly with error term (9) (Model IV). In view of the observed relative frequency, a VAR(1) model is selected by all versions of the AIC criteria and they have the same performance in Model IV. By contrast, Table 4 shows that a modified criterion has clearly hight performance in Model III. 
Table 2

Relative frequency (in \%) of the order selected by various standard and modified versions of the AIC criteria.

\begin{tabular}{|c|c|c|c|c|}
\hline \multirow{2}{*}{$\begin{array}{l}\text { Length } \\
n\end{array}$} & \multirow{2}{*}{$\begin{array}{l}\text { Order } \\
p\end{array}$} & \multicolumn{3}{|c|}{ Criteria Model I } \\
\hline & & AIC & $\mathrm{AICc}$ & $\mathrm{AIC}_{M}$ \\
\hline \multirow{6}{*}{50} & 1 & 84.9 & 91.1 & 90.6 \\
\hline & 2 & 9.3 & 7.4 & 7.8 \\
\hline & 3 & 3.0 & 1.3 & 1.2 \\
\hline & 4 & 0.8 & 0.1 & 0.2 \\
\hline & 5 & 1.1 & 0.1 & 0.2 \\
\hline & 6 & 0.9 & 0.0 & 0.0 \\
\hline \multirow{6}{*}{100} & 1 & 86.9 & 90.4 & 90.9 \\
\hline & 2 & 8.9 & 7.5 & 7.1 \\
\hline & 3 & 2.7 & 1.6 & 1.4 \\
\hline & 4 & 0.7 & 0.3 & 0.4 \\
\hline & 5 & 0.4 & 0.0 & 0.0 \\
\hline & 6 & 0.4 & 0.2 & 0.2 \\
\hline \multirow{6}{*}{200} & 1 & 88.6 & 89.4 & 89.6 \\
\hline & 2 & 6.7 & 7.0 & 6.8 \\
\hline & 3 & 2.8 & 2.4 & 2.4 \\
\hline & 4 & 1.0 & 0.7 & 0.7 \\
\hline & 5 & 0.5 & 0.4 & 0.4 \\
\hline & 6 & 0.4 & 0.1 & 0.1 \\
\hline
\end{tabular}

I: Strong VAR(1) model (10)-(6) 
Table 3

Relative frequency (in \%) of the order selected by various standard and modified versions of the AIC criteria.

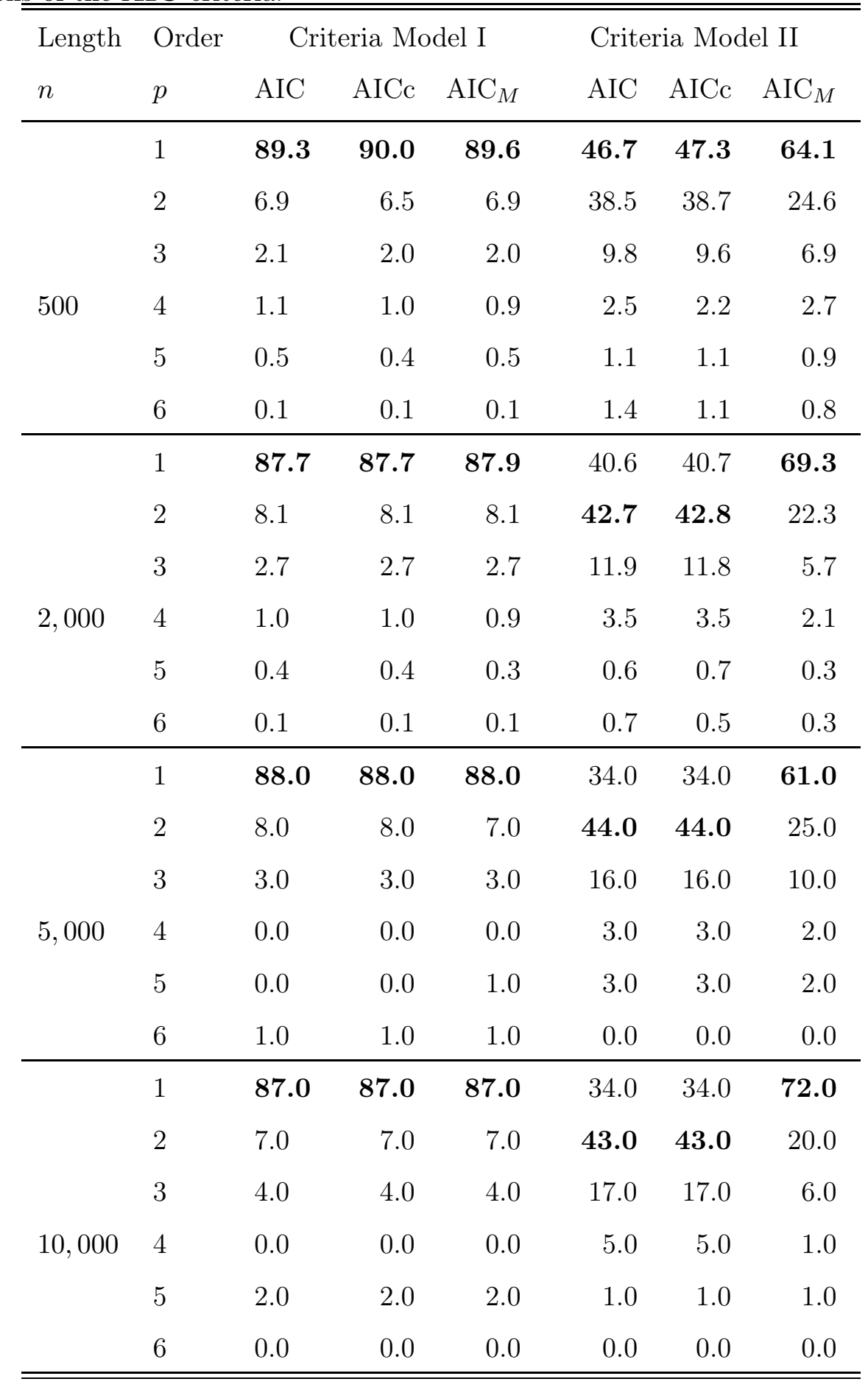

I: Strong VAR(1) model (10)-(6), II: Weak VAR(1) model (10)-(8) 
Table 4

Relative frequency (in \%) of the order selected by various standard and modified versions of the AIC criteria.

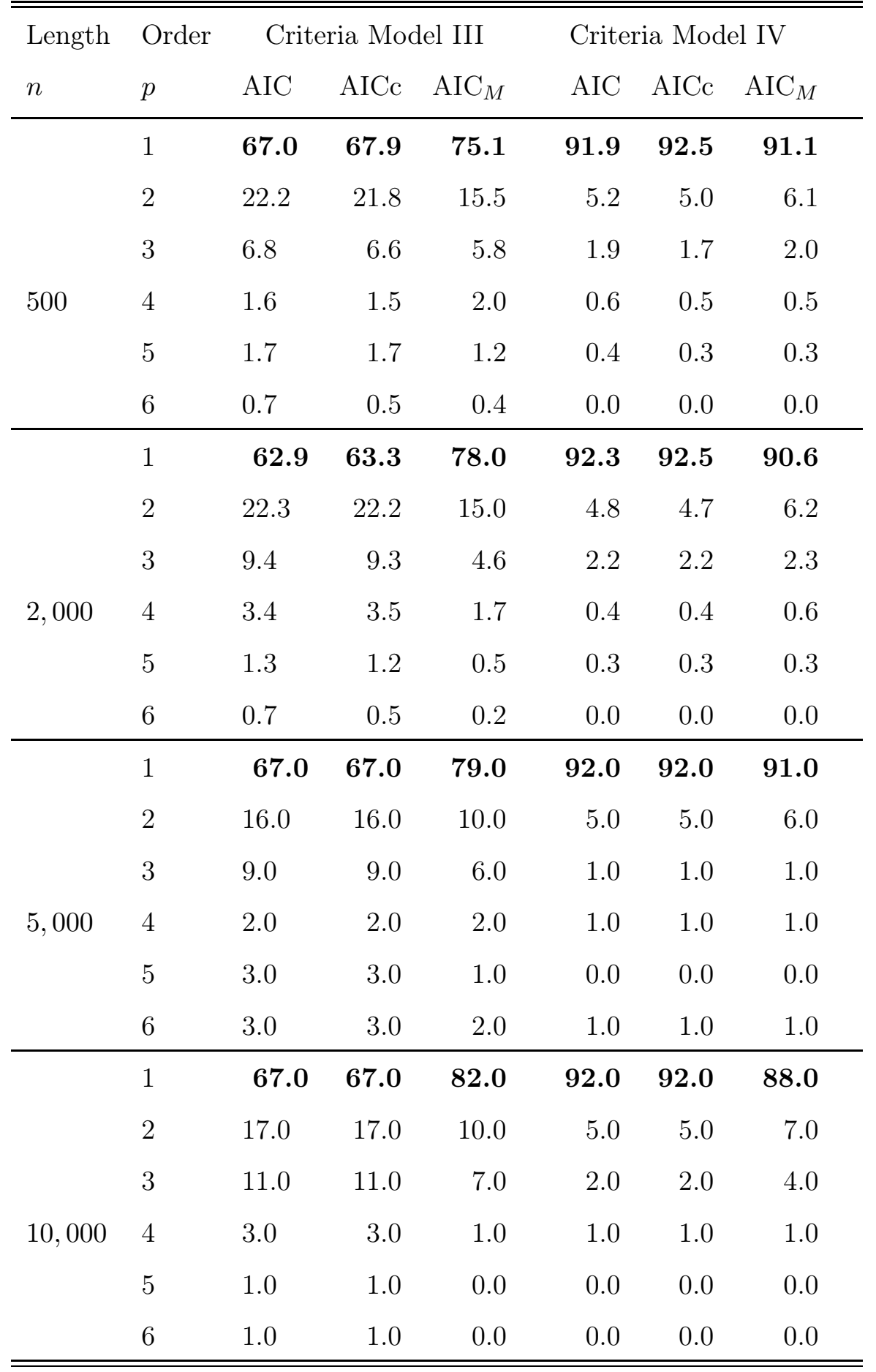

III: Weak VAR(1) model (10)-(7), IV: Weak VAR(1) model (10)-(9) 
Table 5

Modified version of asymptotic probabilities of overfitting by $\ell=d^{2} \ell_{1}$ parameters for bivariate VAR models of various versions of AIC criteria.

\begin{tabular}{llllllll}
\hline \hline \multirow{2}{*}{$\begin{array}{l}\text { Length } \\
n\end{array}$} & Order & \multicolumn{3}{c}{$\mathbf{P}_{W}$ Model I } & \multicolumn{3}{c}{$\mathbf{P}_{W}$ Model II } \\
& $\ell_{1}$ & $\mathbf{P}_{W}^{A I C}$ & $\mathbf{P}_{W}^{A I C c}$ & $\mathbf{P}_{W}^{A I C_{M}}$ & $\mathbf{P}_{W}^{A I C}$ & $\mathbf{P}_{W}^{A I C c}$ & $\mathbf{P}_{W}^{A I C_{M}}$ \\
\hline \multirow{2}{*}{500} & 1 & 0.076 & 0.072 & 0.075 & 0.492 & 0.486 & 0.325 \\
& 2 & 0.040 & 0.037 & 0.039 & 0.370 & 0.359 & 0.221 \\
& 3 & 0.018 & 0.015 & 0.014 & 0.243 & 0.231 & 0.144 \\
& 4 & 0.015 & 0.010 & 0.013 & 0.172 & 0.157 & 0.091 \\
& 5 & 0.003 & 0.002 & 0.002 & 0.109 & 0.099 & 0.059 \\
\hline \multirow{2}{*}{2000} & 1 & 0.101 & 0.101 & 0.100 & 0.557 & 0.557 & 0.283 \\
& 2 & 0.046 & 0.044 & 0.045 & 0.406 & 0.403 & 0.204 \\
& 3 & 0.027 & 0.025 & 0.026 & 0.274 & 0.271 & 0.120 \\
& 4 & 0.014 & 0.013 & 0.012 & 0.172 & 0.168 & 0.077 \\
\hline \hline
\end{tabular}

I: Strong VAR(1) model (10)-(6)

II: Weak VAR(1) model (10)-(8) 
Table 5 displays the modified version of asymptotic probabilities of overfitting by $\ell:=d^{2} \ell_{1}$ parameters for bivariate VAR models of various versions of AIC criteria. Table 5 shows clearly that the $\mathrm{AIC}_{M}$ criterion is not consistent in the weak and strong cases, since his probability of overfitting is not zero. As expected, the asymptotic probabilities of overfitting of the standard versions of the AIC criteria are very strong than the modified criterion in the weak case. By contrast, they are similar in the strong case for all versions of the AIC criteria. The asymptotic probabilities of overfitting of the modified version is decreasing with the sample size $n$.

\section{Conclusion}

The results of Section 4 suggest that the relative frequency of the orders selected by the standard criteria (AIC and $\mathrm{AICc}$ ) and by the modified $\mathrm{AIC}_{M}$ versions are comparable, with a slight advantage to the modified version, in the strong VAR model case. In the weak VAR models cases, the modified version performs better than the standard versions, which often overestimate the order.

\section{Appendix}

Proof of Lemma 1: We have

$$
\begin{aligned}
\Delta(\theta)= & n \log \operatorname{det} \Sigma_{e}+n \operatorname{Tr}\left(\Sigma _ { e } ^ { - 1 } \left\{E e_{1}\left(\theta_{0}\right) e_{1}^{\prime}\left(\theta_{0}\right)+2 E e_{1}\left(\theta_{0}\right)\left\{e_{1}(\theta)-e_{1}\left(\theta_{0}\right)\right\}^{\prime}\right.\right. \\
& \left.\left.+E\left(e_{1}(\theta)-e_{1}\left(\theta_{0}\right)\right)\left(e_{1}(\theta)-e_{1}\left(\theta_{0}\right)\right)^{\prime}\right\}\right) .
\end{aligned}
$$

Now, using the fact that the linear innovation $e_{t}\left(\theta_{0}\right)$ is orthogonal to the linear past (i.e. to the Hilbert space $H_{t-1}$ generated by the linear combinations of the $X_{u}$ for $\left.u<t\right)$, it follows that $E e_{1}\left(\theta_{0}\right)\left\{e_{1}(\theta)-e_{1}\left(\theta_{0}\right)\right\}^{\prime}=0$, since $\left\{e_{t}(\theta)-e_{t}\left(\theta_{0}\right)\right\}$ belongs to the linear past $H_{t-1}$. We thus have

$$
\begin{aligned}
\Delta(\theta)= & n \log \operatorname{det} \Sigma_{e}+n \operatorname{Tr}\left(\Sigma_{e}^{-1} \Sigma_{e 0}\right) \\
& +n \operatorname{Tr}\left\{\Sigma_{e}^{-1} E\left(e_{1}(\theta)-e_{1}\left(\theta_{0}\right)\right)\left(e_{1}(\theta)-e_{1}\left(\theta_{0}\right)\right)^{\prime}\right\} .
\end{aligned}
$$

Moreover

$$
\begin{aligned}
\Delta\left(\theta_{0}\right) & =n \log \operatorname{det} \Sigma_{e 0}+n \operatorname{Tr}\left(\Sigma_{e 0}^{-1} S\left(\theta_{0}\right)\right)=n \log \operatorname{det} \Sigma_{e 0}+n \operatorname{Tr}\left(\Sigma_{e 0}^{-1} \Sigma_{e 0}\right) \\
& =n \log \operatorname{det} \Sigma_{e 0}+n d .
\end{aligned}
$$


Thus, we obtain

$$
\begin{aligned}
\Delta(\theta)-\Delta\left(\theta_{0}\right)= & -n \log \operatorname{det}\left(\Sigma_{e}^{-1} \Sigma_{e 0}\right)-n d+n \operatorname{Tr}\left(\Sigma_{e}^{-1} \Sigma_{e 0}\right) \\
& +n \operatorname{Tr}\left\{\Sigma_{e}^{-1} E\left(e_{1}(\theta)-e_{1}\left(\theta_{0}\right)\right)\left(e_{1}(\theta)-e_{1}\left(\theta_{0}\right)\right)^{\prime}\right\} \\
\geq & -n \log \operatorname{det}\left(\Sigma_{e}^{-1} \Sigma_{e 0}\right)-n d+n \operatorname{Tr}\left(\Sigma_{e}^{-1} \Sigma_{e 0}\right),
\end{aligned}
$$

with equality if and only if $e_{1}(\theta)=e_{1}\left(\theta_{0}\right)$ a.s. Using the elementary inequality $\operatorname{Tr}\left(A^{-1} B\right)-\log \operatorname{det}\left(A^{-1} B\right) \geq \operatorname{Tr}\left(A^{-1} A\right)-\log \operatorname{det}\left(A^{-1} A\right)=d$ for all symmetric positive semi-definite matrices of order $d \times d$, it is easy see that $\Delta(\theta)-\Delta\left(\theta_{0}\right) \geq 0$. The proof is complete.

Justification of (3). Let $J_{11}$ and $I_{11}$ be respectively the upper-left block of the matrices $J$ and $I$, with appropriate size. Recall that

$$
E \Delta\left(\hat{\theta}_{n}\right)=E n \log \operatorname{det} \hat{\Sigma}_{e}+n E \operatorname{Tr}\left(\hat{\Sigma}_{e}^{-1} S\left(\hat{\theta}_{n}\right)\right)
$$

where $\hat{\Sigma}_{e}=n^{-1} \sum_{t=1}^{n} e_{t}\left(\hat{\theta}_{n}\right) e_{t}^{\prime}\left(\hat{\theta}_{n}\right)$. Then the first term on the right-hand side of (11) can be estimated without bias by $n \log \operatorname{det}\left\{n^{-1} \sum_{t=1}^{n} e_{t}\left(\hat{\theta}_{n}\right) e_{t}^{\prime}\left(\hat{\theta}_{n}\right)\right\}$. Hence, only an estimate for the second term needs to be considered. Moreover, in view of (2), a Taylor expansion of $e_{t}(\theta)$ around $\theta_{0}^{(1)}$ yields

$$
e_{t}(\theta)=e_{t}\left(\theta_{0}\right)+\frac{\partial e_{t}\left(\theta_{0}\right)}{\partial \theta^{(1)^{\prime}}}\left(\theta^{(1)}-\theta_{0}^{(1)}\right)+R_{t}
$$

where

$$
R_{t}=\frac{1}{2}\left(\theta^{(1)}-\theta_{0}^{(1)}\right)^{\prime} \frac{\partial^{2} e_{t}\left(\theta^{*}\right)}{\partial \theta^{(1)} \partial \theta^{(1)^{\prime}}}\left(\theta^{(1)}-\theta_{0}^{(1)}\right)=O_{P}\left(\pi^{2}\right),
$$

with $\pi=\left\|\theta^{(1)}-\theta_{0}^{(1)}\right\|$ and $\theta^{*}$ is between $\theta_{0}^{(1)}$ and $\theta^{(1)}$. We then obtain

$$
\begin{aligned}
S(\theta)= & S\left(\theta_{0}\right)+E\left\{\frac{\partial e_{t}\left(\theta_{0}\right)}{\partial \theta^{(1)^{\prime}}}\left(\theta^{(1)}-\theta_{0}^{(1)}\right) e_{t}^{\prime}\left(\theta_{0}\right)\right\}+E R_{t} e_{t}^{\prime}\left(\theta_{0}\right) \\
& +E\left\{e_{t}\left(\theta_{0}\right)\left(\theta^{(1)}-\theta_{0}^{(1)}\right)^{\prime} \frac{\partial e_{t}^{\prime}\left(\theta_{0}\right)}{\partial \theta^{(1)}}\right\}+D\left(\theta^{(1)}\right) \\
& +E R_{t}\left\{\left(\theta^{(1)}-\theta_{0}^{(1)}\right)^{\prime} \frac{\partial e_{t}^{\prime}\left(\theta_{0}\right)}{\partial \theta^{(1)}}\right\}+E e_{t}\left(\theta_{0}\right) R_{t} \\
& +E\left\{\frac{\partial e_{t}\left(\theta_{0}\right)}{\partial \theta^{(1)^{\prime}}}\left(\theta^{(1)}-\theta_{0}^{(1)}\right)\right\} R_{t}+E R_{t}^{2},
\end{aligned}
$$

where

$$
D\left(\theta^{(1)}\right)=E\left\{\frac{\partial e_{t}\left(\theta_{0}\right)}{\partial \theta^{(1)^{\prime}}}\left(\theta^{(1)}-\theta_{0}^{(1)}\right)\left(\theta^{(1)}-\theta_{0}^{(1)}\right)^{\prime} \frac{\partial e_{t}^{\prime}\left(\theta_{0}\right)}{\partial \theta^{(1)}}\right\}
$$


Using the orthogonality between $e_{t}\left(\theta_{0}\right)$ and any linear combination of the past values of $e_{t}\left(\theta_{0}\right)$ (in particular $\partial e_{t}\left(\theta_{0}\right) / \partial \theta^{\prime}$ and $\partial^{2} e_{t}\left(\theta_{0}\right) / \partial \theta \partial \theta^{\prime}$ ), and the fact that $E e_{t}\left(\theta_{0}\right)=0$, we have

$$
S(\theta)=S\left(\theta_{0}\right)+D\left(\theta^{(1)}\right)+O\left(\pi^{4}\right)=\Sigma_{e 0}+D\left(\theta^{(1)}\right)+O\left(\pi^{4}\right),
$$

where $\Sigma_{e 0}=\Sigma_{e}\left(\theta_{0}\right)$. Thus, we can write the expected discrepancy quantity in (11) as

$$
\begin{aligned}
E \Delta\left(\hat{\theta}_{n}\right)= & E n \log \operatorname{det} \hat{\Sigma}_{e}+n E \operatorname{Tr}\left(\hat{\Sigma}_{e}^{-1} \Sigma_{e 0}\right)+n E \operatorname{Tr}\left(\hat{\Sigma}_{e}^{-1} D\left(\hat{\theta}_{n}^{(1)}\right)\right) \\
& +n E\left\{\operatorname{Tr}\left(\hat{\Sigma}_{e}^{-1}\right) O_{P}\left(\frac{1}{n^{2}}\right)\right\}
\end{aligned}
$$

As in the classical multivariate regression model, we deduce

$$
\Sigma_{e 0} \approx \frac{n}{n-d(p+q)} E\left\{\hat{\Sigma}_{e}\right\}=\frac{d n}{d n-k_{1}} E\left\{\hat{\Sigma}_{e}\right\}, \quad \text { where } \quad k_{1}=d^{2}(p+q)
$$

Thus, using the last approximation and from the consistency of $\hat{\Sigma}_{e}$, we obtain

$$
E\left\{\hat{\Sigma}_{e}^{-1}\right\} \approx\left\{E \hat{\Sigma}_{e}\right\}^{-1} \approx n d\left(n d-k_{1}\right)^{-1} \Sigma_{e 0}^{-1}
$$

An alternative to (13) is to use a slightly more accurate result, as in Hurvich and Tsai (1993), by treating $n \hat{\Sigma}_{e}$ as having a asymptotic Wishart distribution ${ }^{1}$ with matrix $\Sigma_{e 0}$ and $n-d(p+q)$ degrees of freedom, so that $E\left\{\hat{\Sigma}_{e}^{-1}\right\} \approx$ $n /[n-d(p+q)-d-1] \Sigma_{e 0}^{-1}$. See Wei (1994, p. 406) and Anderson (2003, p. 296) for these results.

Using the elementary property on the trace, we have

$$
\begin{aligned}
\operatorname{Tr}\left\{\Sigma_{e}^{-1}(\theta) D\left(\theta_{n}^{(1)}\right)\right\} & =\operatorname{Tr}\left(\Sigma_{e}^{-1}(\theta) E\left\{\frac{\partial e_{t}\left(\theta_{0}\right)}{\partial \theta^{(1)^{\prime}}}\left(\theta^{(1)}-\theta_{0}^{(1)}\right)\left(\theta^{(1)}-\theta_{0}^{(1)}\right)^{\prime} \frac{\partial e_{t}^{\prime}\left(\theta_{0}\right)}{\partial \theta^{(1)}}\right\}\right) \\
& =E\left(\operatorname{Tr}\left\{\frac{\partial e_{t}^{\prime}\left(\theta_{0}\right)}{\partial \theta^{(1)}} \Sigma_{e}^{-1}(\theta) \frac{\partial e_{t}\left(\theta_{0}\right)}{\partial \theta^{(1)^{\prime}}}\left(\theta^{(1)}-\theta_{0}^{(1)}\right)^{\prime}\left(\theta^{(1)}-\theta_{0}^{(1)}\right)\right\}\right) \\
& =\operatorname{Tr}\left(E\left\{\frac{\partial e_{t}^{\prime}\left(\theta_{0}\right)}{\partial \theta^{(1)}} \Sigma_{e}^{-1}(\theta) \frac{\partial e_{t}\left(\theta_{0}\right)}{\partial \theta^{(1)^{\prime}}}\right\}\left(\theta^{(1)}-\theta_{0}^{(1)}\right)^{\prime}\left(\theta^{(1)}-\theta_{0}^{(1)}\right)\right) .
\end{aligned}
$$

Now, using (2), (13) and the last equality, the third term in (12) becomes

1 The Wishart distribution arises in a natural way as a matrix generalization of the chi-square distribution. 


$$
\begin{aligned}
E \operatorname{Tr}\left\{\hat{\Sigma}_{e}^{-1} D\left(\hat{\theta}_{n}^{(1)}\right)\right\}= & \frac{1}{n} \operatorname{Tr}\left(E\left\{\frac{\partial e_{t}^{\prime}\left(\theta_{0}\right)}{\partial \theta^{(1)}} \hat{\Sigma}_{e}^{-1} \frac{\partial e_{t}\left(\theta_{0}\right)}{\partial \theta^{(1)^{\prime}}}\right\}\right. \\
& \left.\operatorname{En}\left(\hat{\theta}_{n}^{(1)}-\theta_{0}^{(1)}\right)^{\prime}\left(\hat{\theta}_{n}^{(1)}-\theta_{0}^{(1)}\right)\right) \\
& =\frac{d}{n d-k_{1}} \operatorname{Tr}\left(E\left\{\frac{\partial e_{t}^{\prime}\left(\theta_{0}\right)}{\partial \theta^{(1)}} \Sigma_{e 0}^{-1} \frac{\partial e_{t}\left(\theta_{0}\right)}{\partial \theta^{(1)^{\prime}}}\right\} J_{11}^{-1} I_{11} J_{11}^{-1}\right) \\
& =\frac{d}{2\left(n d-k_{1}\right)} \operatorname{Tr}\left(I_{11} J_{11}^{-1}\right),
\end{aligned}
$$

where $J_{11}=2 E\left\{\partial e_{t}^{\prime}\left(\theta_{0}\right) / \partial \theta^{(1)} \Sigma_{e 0}^{-1} \partial e_{t}\left(\theta_{0}\right) / \partial \theta^{(1)^{\prime}}\right\}$ (see Theorem 3 in BMF). Thus, using (13), the second term in (11) becomes

$$
\begin{aligned}
E \operatorname{Tr}\left(\hat{\Sigma}_{e}^{-1} S\left(\hat{\theta}_{n}\right)\right)= & E \operatorname{Tr}\left(\hat{\Sigma}_{e}^{-1} \Sigma_{e 0}\right)+E \operatorname{Tr}\left\{\hat{\Sigma}_{e}^{-1} D\left(\hat{\theta}_{n}^{(1)}\right)\right\} \\
& +E\left\{\operatorname{Tr}\left(\hat{\Sigma}_{e}^{-1}\right) O_{P}\left(\frac{1}{n^{2}}\right)\right\} \\
= & \frac{n d}{n d-k_{1}} \operatorname{Tr}\left(\Sigma_{e 0}^{-1} \Sigma_{e 0}\right)+\frac{d}{2\left(n d-k_{1}\right)} \operatorname{Tr}\left(I_{11} J_{11}^{-1}\right)+O\left(\frac{1}{n^{2}}\right) \\
= & \frac{n d^{2}}{n d-k_{1}}+\frac{d}{2\left(n d-k_{1}\right)} \operatorname{Tr}\left(I_{11} J_{11}^{-1}\right)+O\left(\frac{1}{n^{2}}\right) .
\end{aligned}
$$

Therefore, using the last equality in (11), we deduce an approximately unbiased estimator of $E \Delta\left(\hat{\theta}_{n}\right)$ given by

$$
\mathrm{AIC}_{M}=n \log \operatorname{det} \hat{\Sigma}_{e}+\frac{n^{2} d^{2}}{n d-k_{1}}+\frac{n d}{2\left(n d-k_{1}\right)} \operatorname{Tr}\left(\hat{I}_{11, n} \hat{J}_{11, n}^{-1}\right)
$$

where $\hat{J}_{11, n}$ and $\hat{I}_{11, n}$ are respectively consistent estimators of the matrice $J_{11}$ and $I_{11}$ defined in Section 4 of BMF. The justification is complete.

Proof of Proposition 1: Using a Taylor expansion of the quasi log-likelihood, we obtain

$$
-2 \log \mathrm{L}_{n}\left(\theta_{0}\right)=-2 \log \mathrm{L}_{n}\left(\hat{\theta}_{n}\right)+\frac{n}{2}\left(\hat{\theta}_{n}^{(1)}-\theta_{0}^{(1)}\right)^{\prime} J_{11}\left(\hat{\theta}_{n}^{(1)}-\theta_{0}^{(1)}\right)+o_{P}(1) .
$$

Taking the expectation (under the true model) of both sides, and in view of (2) we shown that

$$
\begin{aligned}
E_{X} n\left(\hat{\theta}_{n}^{(1)}-\theta_{0}^{(1)}\right)^{\prime} J_{11}\left(\hat{\theta}_{n}^{(1)}-\theta_{0}^{(1)}\right) & =\operatorname{Tr}\left\{J_{11} E_{X} n\left(\hat{\theta}_{n}^{(1)}-\theta_{0}^{(1)}\right)^{\prime}\left(\hat{\theta}_{n}^{(1)}-\theta_{0}^{(1)}\right)\right\} \\
& \rightarrow \operatorname{Tr}\left(I_{11} J_{11}^{-1}\right),
\end{aligned}
$$

we then obtain $a_{1}=2^{-1} \operatorname{Tr}\left(I_{11} J_{11}^{-1}\right)+o(1)$. 
Now a Taylor expansion of the discrepancy yields

$$
\begin{aligned}
\Delta\left(\hat{\theta}_{n}\right)= & \Delta\left(\theta_{0}\right)+\left.\left(\hat{\theta}_{n}^{(1)}-\theta_{0}^{(1)}\right)^{\prime} \frac{\partial \Delta(\theta)}{\partial \theta^{(1)}}\right|_{\theta=\theta_{0}} \\
& +\left.\frac{1}{2}\left(\hat{\theta}_{n}^{(1)}-\theta_{0}^{(1)}\right)^{\prime} \frac{\partial^{2} \Delta(\theta)}{\partial \theta^{(1)} \partial \theta^{(1)^{\prime}}}\right|_{\theta=\theta_{0}}\left(\hat{\theta}_{n}^{(1)}-\theta_{0}^{(1)}\right)+o_{P}(1) \\
= & \Delta\left(\theta_{0}\right)+\frac{n}{2}\left(\hat{\theta}_{n}^{(1)}-\theta_{0}^{(1)}\right)^{\prime} J_{11}\left(\hat{\theta}_{n}^{(1)}-\theta_{0}^{(1)}\right)+o_{P}(1)
\end{aligned}
$$

assuming that the discrepancy is smooth enough, and that we can take its derivatives under the expectation sign. We then deduce that

$$
E_{Y}-2 \log \mathrm{L}_{n}\left(\hat{\theta}_{n}\right)=E_{X} \Delta\left(\hat{\theta}_{n}\right)=E_{X} \Delta\left(\theta_{0}\right)+\frac{1}{2} \operatorname{Tr}\left(I_{11} J_{11}^{-1}\right)+o(1)
$$

which shows that $a_{2}$ is equivalent to $a_{1}$. The proof is complete.

Proof of Proposition 2: We denote by $|A|$, the determinant of the matrix $A$. The probability that the $\mathrm{AIC}_{M}$ criterion selects the overfitted model is

$$
\begin{aligned}
& P\left\{\operatorname{AIC}_{M}\left(k_{1}^{\prime}\right)<\operatorname{AIC}_{M}\left(k_{1}\right)\right\}=P\left\{n \log \left|\hat{\Sigma}_{e}\left(k_{1}^{\prime}\right)\right|+\frac{n^{2} d^{2}}{n d-k_{1}^{\prime}}+\frac{n d c_{k_{1}^{\prime}}}{2\left(n d-k_{1}^{\prime}\right)}\right. \\
& \left.<n \log \left|\hat{\Sigma}_{e}\left(k_{1}\right)\right|+\frac{n^{2} d^{2}}{n d-k_{1}}+\frac{n d c_{k_{1}}}{2\left(n d-k_{1}\right)}\right\} \\
& =P\left\{\operatorname{AIC}_{M}\left(k_{1}+\ell\right)<\operatorname{AIC}_{M}\left(k_{1}\right)\right\} \\
& =P\left\{n \log \left\{\frac{\left|n \hat{\Sigma}_{e}\left(k_{1}+\ell\right)\right|}{\left|n \hat{\Sigma}_{e}\left(k_{1}\right)\right|}\right\}<\frac{n^{2} d^{2}}{n d-k_{1}}\right. \\
& +\frac{n d c_{k_{1}}}{2\left(n d-k_{1}\right)}-\frac{n^{2} d^{2}}{n d-\left(k_{1}+\ell\right)} \\
& \left.-\frac{n d\left(c_{k_{1}}+c_{\ell}\right)}{2\left[n d-\left(k_{1}+\ell\right)\right]}\right\} \\
& =P\left\{n \log \left\{\frac{\left|n \hat{\Sigma}_{e}\left(k_{1}+\ell\right)\right|}{\left|n \hat{\Sigma}_{e}\left(k_{1}\right)\right|}\right\}\right. \\
& <\frac{-n^{2} \ell d^{2}}{\left(n d-k_{1}\right)\left[n d-\left(k_{1}+\ell\right)\right]} \\
& \left.+\frac{n d\left(k_{1} c_{\ell}-\ell c_{k_{1}}\right)-n^{2} d^{2} c_{\ell}}{2\left(n d-k_{1}\right)\left[n d-\left(k_{1}+\ell\right)\right]}\right\} \text {. }
\end{aligned}
$$

Let $q_{1}=k_{1} / d$ and $q_{2}=\left(k_{1}+\ell\right) / d$, we denote 


$$
\frac{\left|n \hat{\Sigma}_{e}\left(k_{1}+\ell\right)\right|}{\left|n \hat{\Sigma}_{e}\left(k_{1}\right)\right|}=\frac{\left|n \hat{\Sigma}_{e}\left(k_{1}+\ell\right)\right|}{\left|n \hat{\Sigma}_{e}\left(k_{1}+\ell\right)+n\left\{\hat{\Sigma}_{e}\left(k_{1}\right)-\hat{\Sigma}_{e}\left(k_{1}+\ell\right)\right\}\right|} \sim U_{d, \ell, n-q_{2}},
$$

where $U_{d, \ell, n-q_{2}}$ is the U-statistic (see Anderson, 2003, chap. 8), a generalized version of the F-statistic used for the univariate case. From Theorem 3.2.15 in Muirhead (1982, p. 100), the distribution of the determinants $\left|n \hat{\Sigma}_{e}\left(k_{1}\right)\right|$ and $\left|n \hat{\Sigma}_{e}\left(k_{1}+\ell\right)\right|$ are respectively the product of independent $\chi^{2}$ random variables,

$$
\frac{\left|n \hat{\Sigma}_{e}\left(k_{1}\right)\right|}{\left|\Sigma_{e 0}\right|} \sim \prod_{i=1}^{d} \chi_{n-q_{1}-i+1}^{2} \quad \text { and } \quad \frac{\left|n \hat{\Sigma}_{e}\left(k_{1}+\ell\right)\right|}{\left|\Sigma_{e 0}\right|} \sim \prod_{i=1}^{d} \chi_{n-q_{2}-i+1}^{2} .
$$

Note that in view of Theorem 7.3.2 (see Anderson, 2003, p. 260) $n\left\{\hat{\Sigma}_{e}\left(k_{1}\right)-\hat{\Sigma}_{e}\left(k_{1}+\ell\right)\right\} \sim \mathrm{W}_{d}\left(\ell / d, \Sigma_{e 0}\right)$, where the subscript on $\mathrm{W}$ denoting the size of the matrix $\Sigma_{e 0}$. Using the previous results and Lemma 8.4.2 (see Anderson, 2003, p. 305), it follows that the distribution of the ratio $\left|n \hat{\Sigma}_{e}\left(k_{1}+\ell\right)\right| /\left|n \hat{\Sigma}_{e}\left(k_{1}\right)\right|$ is the multivariate Beta $_{d}$ distribution $^{2}$ i.e. the product of independents Beta distributions (see Anderson, 2003, Section 5.2):

$$
\frac{\left|n \hat{\Sigma}_{e}\left(k_{1}+\ell\right)\right|}{\left|n \hat{\Sigma}_{e}\left(k_{1}\right)\right|} \sim \prod_{i=1}^{d} \operatorname{Beta}\left(\frac{n-q_{2}-i+1}{2}, \frac{\ell}{2 d}\right) .
$$

Expressed in terms of independent $\chi^{2}$, we obtain

$$
\left\{\frac{\left|n \hat{\Sigma}_{e}\left(k_{1}+\ell\right)\right|}{\left|n \hat{\Sigma}_{e}\left(k_{1}\right)\right|}\right\}^{-1}=\frac{\left|n \hat{\Sigma}_{e}\left(k_{1}\right)\right|}{\left|n \hat{\Sigma}_{e}\left(k_{1}+\ell\right)\right|} \sim \prod_{i=1}^{d}\left(1+\frac{\chi_{\ell / d}^{2}}{\chi_{n-q_{2}-i+1}^{2}}\right) .
$$

Thus the probability of overfitting for $\mathrm{AIC}_{M}$ criterion can be rewrite as

$$
\begin{aligned}
P\left\{\operatorname{AIC}_{M}\left(k_{1}+\ell\right)<\operatorname{AIC}_{M}\left(k_{1}\right)\right\}= & P\left\{-n \sum_{i=1}^{d} \log \left(1+\frac{\chi_{\ell / d}^{2}}{\chi_{n-q_{2}-i+1}^{2}}\right)\right. \\
& <\frac{-n^{2} \ell d^{2}}{\left(n d-k_{1}\right)\left[n d-\left(k_{1}+\ell\right)\right]} \\
& \left.+\frac{n d\left(k_{1} c_{\ell}-\ell c_{k_{1}}\right)-n^{2} d^{2} c_{\ell}}{2\left(n d-k_{1}\right)\left[n d-\left(k_{1}+\ell\right)\right]}\right\} .
\end{aligned}
$$

Recall that, $\log (1+x) \simeq x$ for small value of $|x|$. Using the fact that $\chi_{n-q_{2}-i+1}^{2} / n \rightarrow 1$ a.s. as $n \rightarrow \infty$ for $k_{1}$, $\ell$ fixed and $1 \leq i \leq d$; it follows that

$\overline{2}$ The multivariate beta distribution generalizes the usual beta distribution in much the same way that the Wishart distribution generalizes the $\chi^{2}$ distribution. 


$$
\begin{aligned}
n \sum_{i=1}^{d} \log \left(1+\frac{\chi_{\ell / d}^{2}}{\chi_{n-q_{2}-i+1}^{2}}\right) & =n \sum_{i=1}^{d} \log \left(1+\frac{(1 / n) \chi_{\ell / d}^{2}}{(1 / n) \chi_{n-q_{2}-i+1}^{2}}\right) \\
& \rightarrow n \sum_{i=1}^{d} \frac{(1 / n) \chi_{\ell / d}^{2}}{(1 / n) \chi_{n-q_{2}-i+1}^{2}} \rightarrow \sum_{i=1}^{d} \chi_{\ell / d}^{2}=\chi_{\ell}^{2} .
\end{aligned}
$$

Note that, as $n \rightarrow \infty$, for $k_{1}, \ell$ and $d$ fixed, we have

$$
\begin{aligned}
& \frac{-n^{2} \ell d^{2}}{\left(n d-k_{1}\right)\left[n d-\left(k_{1}+\ell\right)\right]}+\frac{n d\left(k_{1} c_{\ell}-\ell c_{k_{1}}\right)-n^{2} d^{2} c_{\ell}}{2\left(n d-k_{1}\right)\left[n d-\left(k_{1}+\ell\right)\right]} \\
= & \frac{-2 n^{2} \ell d^{2}+n d\left(k_{1} c_{\ell}-\ell c_{k_{1}}\right)-n^{2} d^{2} c_{\ell}}{2\left(n d-k_{1}\right)\left[n d-\left(k_{1}+\ell\right)\right]} \rightarrow-\frac{2 \ell+c_{\ell}}{2} .
\end{aligned}
$$

In view of (14) and (15), we deduce the following asymptotic probability of overfitting

$$
P\left\{\operatorname{AIC}_{M}\left(k_{1}+\ell\right)<\operatorname{AIC}_{M}\left(k_{1}\right)\right\}=P\left\{\chi_{\ell}^{2}>\frac{2 \ell+c_{\ell}}{2}\right\} .
$$

The proof is complete. 


\section{References}

Akaike, H. (1973) Information theory and an extension of the maximum likelihood principle. In 2nd International Symposium on Information Theory, Eds. B. N. Petrov and F. Csáki, pp. 267-281. Budapest: Akadémia Kiado.

Anderson, T. W. (2003) An Introduction to Multivariate Statistical Analysis 3nd Edn. New Jersey, Wiley.

Boubacar Mainassara, Y. (2009a) Multivariate portmanteau test for structural VARMA models with uncorrelated but non-independent error terms. MPRA Working Papers, http://mpra.ub.uni-muenchen.de/23371/

Boubacar Mainassara, Y. (2009b) Estimating the asymptotic variance matrix of structural VARMA models with uncorrelated but non-independent error terms. Working Papers, http://perso.univlille3.fr/ yboubacarmai/yac/VARMAEstCOV2.pdf

Boubacar Mainassara, Y. and Francq, C. (2009) Estimating structural VARMA models with uncorrelated but non-independent error terms. MPRA Working Papers, http://mpra.ub.uni-muenchen.de/15141/

Dufour, J-M., and Pelletier, D. (2005) Practical methods for modelling weak VARMA processes: identification, estimation and specification with a macroeconomic application. Technical report, Département de sciences économiques and CIREQ, Université de Montréal, Montréal, Canada.

Findley, D.F. (1993) The overfitting principles supporting AIC, Statistical Research Division Report RR 93/04, Bureau of the Census.

Francq, C. and Raïssi, H. (2007) Multivariate Portmanteau Test for Autoregressive Models with Uncorrelated but Nonindependent Errors, Journal of Time Series Analysis 28, 454-470.

Francq, C., Roy, R. and Zakoïan, J-M. (2005) Diagnostic checking in ARMA Models with Uncorrelated Errors, Journal of the American Statistical Association 100, 532-544.

Francq, and Zakoïan, J-M. (1998) Estimating linear representations of nonlinear processes, Journal of Statistical Planning and Inference 68, 145165.

Francq, and Zakoïan, J-M. (2000) Covariance matrix estimation of mixing weak ARMA models, Journal of Statistical Planning and Inference 83, 369-394.

Francq, and Zakoïan, J-M. (2005) Recent results for linear time series models with non independent innovations. In Statistical Modeling and Analysis for Complex Data Problems, Chap. 12 (eds P. DuChesne and B. RÉmillard). New York: Springer Verlag, 241-265.

Francq, and Zakoïan, J-M. (2007) HAC estimation and strong linearity testing in weak ARMA models, Journal of Multivariate Analysis 98, 114144.

Hannan, E. J. and Rissanen (1982) Recursive estimation of mixed of Autoregressive Moving Average order, Biometrika 69, 81-94. 
Hurvich, C. M. and Tsai, C-L. (1989) Regression and time series model selection in small samples. Biometrika 76, 297-307.

Hurvich, C. M. and Tsai, C-L. (1993) A corrected Akaike information criterion for vector autoregressive model selection. Journal of Time Series Analysis 14, 271-279.

Lütkepohl, H. (1993) Introduction to multiple time series analysis. Springer Verlag, Berlin.

Lütkepohl, H. (2005) New introduction to multiple time series analysis. Springer Verlag, Berlin.

Magnus, J.R. and H. Neudecker (1988) Matrix Differential Calculus with Application in Statistics and Econometrics. New-York, Wiley.

Muirhead, R.J. (1982) Aspects of Multivariate Statistical Theory. Wiley, New-York, Wiley.

Reinsel, G. C. (1997) Elements of multivariate time series Analysis. Second edition. Springer Verlag, New York.

Romano, J. L. and Thombs, L. A. (1996) Inference for autocorrelations under weak assumptions, Journal of the American Statistical Association 91, 590-600.

Wei, W.H. (1994) Time Series Analysis: Univariate and Multivariate Methods 2nd Edn. New York, Addison-Wesley. 
Selection of weak VARMA models by modified Akaike's information criteria: Complementary simulations results that are not submitted for publication

\section{A General multivariate linear regression model}

Now we need to recall several results concerning general multivariate linear regression models.

Let $Z_{t}=\left(Z_{1 t}, \ldots, Z_{d t}\right)^{\prime}$ be a $d$-dimensional random vector of response variables, $X_{t}=\left(X_{1 t}, \ldots, X_{k t}\right)^{\prime}$ be a $k$-dimensional input variables and $B=$ $\left(\beta_{1}, \ldots, \beta_{d}\right)$ be a $k \times d$ matrix. We consider a multivariate linear model of the form $Z_{i t}=X_{t}^{\prime} \beta_{i}+\epsilon_{i t}, i=1, \ldots, d$, or $Z_{t}^{\prime}=X_{t}^{\prime} B+\epsilon_{t}^{\prime}, t=1, \ldots, n$, where the $\epsilon_{t}=\left(\epsilon_{1 t}, \ldots, \epsilon_{d t}\right)^{\prime}$ are uncorrelated and identically distributed random vectors with variance $\Sigma=E \epsilon_{t} \epsilon_{t}^{\prime}$. The $i$-th column of $B\left(i . e . \beta_{i}\right)$ is the vector of regression coefficients for the $i$-th response variable. Now, given the $n$ observations $Z_{1}, \ldots, Z_{n}$ and $X_{1}, \ldots, X_{n}$, we define the $n \times d$ data matrix $\mathbf{Z}=\left(Z_{1}, \ldots, Z_{n}\right)^{\prime}$, the $n \times k$ matrix $\mathbf{X}=\left(X_{1}, \ldots, X_{n}\right)^{\prime}$ and the $n \times d$ matrix $\varepsilon=\left(\epsilon_{1}, \ldots, \epsilon_{n}\right)^{\prime}$. Then, we have the multivariate linear model $\mathbf{Z}=\mathbf{X} B+\varepsilon$. Now, it is well known that the QMLE of $B$ is the same as the LSE and, hence, is given by

$$
\hat{B}=\left(\mathbf{X}^{\prime} \mathbf{X}\right)^{-1} \mathbf{X}^{\prime} \mathbf{Z}, \quad \text { that is, } \quad \hat{\beta}_{i}=\left(\mathbf{X}^{\prime} \mathbf{X}\right)^{-1} \mathbf{X}^{\prime} \mathbf{Z}_{i}, \quad i=1, \ldots, d,
$$

where $\mathbf{Z}_{i}=\left(Z_{i 1}, \ldots, Z_{i n}\right)^{\prime}$ is the $i$-th column of $\mathbf{Z}$. We also have

$$
\hat{\varepsilon}:=\mathbf{Z}-\mathbf{X} \hat{B}=M_{\mathbf{X}} \mathbf{Z}=\varepsilon-\mathbf{X}\left(\mathbf{X}^{\prime} \mathbf{X}\right)^{-1} \mathbf{X}^{\prime} \varepsilon=M_{\mathbf{X}} \varepsilon,
$$

where $M_{\mathbf{X}}=I_{n}-\mathbf{X}\left(\mathbf{X}^{\prime} \mathbf{X}\right)^{-1} \mathbf{X}^{\prime}$ is a projection matrix. The usual unbiased estimator of the error covariance matrix $\Sigma$ is

$$
\begin{aligned}
\Sigma^{*} & =\frac{1}{n-k} \hat{\varepsilon}^{\prime} \hat{\varepsilon}=\frac{1}{n-k}(\mathbf{Z}-\mathbf{X} \hat{B})^{\prime}(\mathbf{Z}-\mathbf{X} \hat{B}) \\
& =\frac{1}{n-k} \sum_{t=1}^{n}\left(Z_{t}-\hat{B}^{\prime} X_{t}\right)\left(Z_{t}-\hat{B}^{\prime} X_{t}\right)^{\prime}
\end{aligned}
$$

or $\Sigma^{*}=(n-k)^{-1} \sum_{t=1}^{n} \hat{\epsilon}_{t} \hat{\epsilon}_{t}^{\prime}$, where the $\hat{\epsilon}_{t}=Z_{t}-\hat{B}^{\prime} X_{t}$ are the residual vectors. Note that the gaussian quasi-likelihood is given by

$$
\mathrm{L}_{n}(B, \Sigma ; \mathbf{Z})=\frac{1}{(2 \pi)^{d / 2} \sqrt{\operatorname{det} \Sigma_{e}}} \exp \left\{-\frac{1}{2} \sum_{t=1}^{n}\left(Z_{t}-B^{\prime} X_{t}\right)^{\prime} \Sigma^{-1}\left(Z_{t}-B^{\prime} X_{t}\right)\right\},
$$


whose maximization shows that the QMLE of $B$ is equal to $\hat{B}$ and that of $\Sigma$ is $\hat{\Sigma}:=n^{-1} \sum_{t=1}^{n} \hat{\epsilon}_{t} \hat{\epsilon}_{t}^{\prime}=(n-k) n^{-1} \Sigma^{*}$. Because $\Sigma^{*}$ is an unbiased estimator of

the matrix $\Sigma$, by definition we have $E\left\{\Sigma^{*}\right\}=\Sigma$, we then deduce that

$$
\frac{n}{n-k} E\{\hat{\Sigma}\}=\frac{1}{n-k} E \hat{\varepsilon}^{\prime} \hat{\varepsilon}=\frac{1}{n-k} E \varepsilon^{\prime} M_{\mathbf{X}} \varepsilon=\Sigma .
$$

\section{B Kullback-Leibler discrepancy}

This Section presents the definition and mains properties of the KullbackLeibler divergence.

Assume that, with respect to a $\sigma$-finite measure $\mu$, the true density of the observations $X=\left(X_{1}, \ldots, X_{n}\right)$ is $f_{0}$, and that some candidate model $m$ gives a density $f_{m}\left(\cdot, \theta_{m}\right)$ to the observations, where $\theta_{m}$ is a $k_{m}$-dimensional parameter. The discrepancy between the candidate and the true models can be measured by the Kullback-Leibler divergence (or information)

$$
d\left\{f_{m}\left(\cdot, \theta_{m}\right) \mid f_{0}\right\}=E_{f_{0}} \log \frac{f_{0}(X)}{f_{m}\left(X, \theta_{m}\right)}=E_{f_{0}} \log f_{0}(X)+\frac{1}{2} \Delta\left\{f_{m}\left(\cdot, \theta_{m}\right) \mid f_{0}\right\}
$$

where

$$
\Delta\left\{f_{m}\left(\cdot, \theta_{m}\right) \mid f_{0}\right\}=-2 E_{f_{0}} \log f_{m}\left(X, \theta_{m}\right)=-2 \int\left\{\log f_{m}\left(x, \theta_{m}\right)\right\} f_{0}(x) \mu(d x)
$$

is sometimes called the Kullback-Leibler contrast (or the discrepancy between the approximating and the true models). Using the Jensen inequality, we have

$$
\begin{aligned}
d\left\{f_{m}\left(\cdot, \theta_{m}\right) \mid f_{0}\right\} & =-\int \log \frac{f_{m}\left(x, \theta_{m}\right)}{f_{0}(x)} f_{0}(x) \mu(d x) \\
& \geq-\log \int \frac{f_{m}\left(x, \theta_{m}\right)}{f_{0}(x)} f_{0}(x) \mu(d x)=0
\end{aligned}
$$

with equality if and only if $f_{m}\left(\cdot, \theta_{m}\right)=f_{0}$. This is the main property of the Kullback-Leibler divergence. Minimizing $d\left\{f_{m}\left(\cdot, \theta_{m}\right) \mid f_{0}\right\}$ with respect to $f_{m}\left(\cdot, \theta_{m}\right)$ is equivalent to minimizing the contrast $\Delta\left\{f_{m}\left(\cdot, \theta_{m}\right) \mid f_{0}\right\}$. Let

$$
\theta_{0, m}=\arg \inf _{\theta_{m}} d\left\{f_{m}\left(\cdot, \theta_{m}\right) \mid f_{0}\right\}=\arg \inf _{\theta_{m}}-2 E \log f_{m}\left(X, \theta_{m}\right)
$$

be an optimal parameter for the model $m$ corresponding to the density $f_{m}\left(\cdot, \theta_{m}\right)$ (assuming that such a parameter exists). We estimate this optimal 
parameter by QMLE $\hat{\theta}_{n, m}$.

\section{Strong and weak VARMA case}

In this Section, we presents the simulations results on the VARMA model in echelon form. We simulated $N$ independent trajectories of different sizes of a bivariate $\operatorname{VARMA}(1,1)$ model in echelon form or, more precisely, an $\operatorname{ARMA}_{E}(0,1)$, with the strong Gaussian and weak noise above-mentioned. We took $N=1,000$ when the sample size $n \leq 2000$ and $N=1,00$ in the opposite case. For each of these $N$ replications of both models, we have 9 candidates models (i.e. $\operatorname{VARMA}(1,1), \operatorname{VARMA}(2,2), \operatorname{VARMA}(2,1), \operatorname{VARMA}(1,2)$, $\operatorname{VARMA}(1,3), \operatorname{VARMA}(3,1), \operatorname{VARMA}(3,2), \operatorname{VARMA}(2,3)$ and $\operatorname{VARMA}(3,3)$ models). These candidates models are constrained in echelon form (i.e. an $\operatorname{ARMA}_{E}(0, k)$ for $\left.k=1,2,3\right)$. The quasi-maximum likelihood method was used to fit candidates bivariate VARMA models and standard and modified versions of AIC criteria were used to select among the candidates models. To generate the strong and weak $\operatorname{VARMA}(1,1)$ model, we consider the bivariate model of the form

$$
\begin{aligned}
\left(\begin{array}{l}
X_{1, t} \\
X_{2, t}
\end{array}\right)= & \left(\begin{array}{cc}
0 & 0 \\
0 & 0.225
\end{array}\right)\left(\begin{array}{l}
X_{1, t-1} \\
X_{2, t-1}
\end{array}\right)+\left(\begin{array}{c}
\epsilon_{1, t} \\
\epsilon_{2, t}
\end{array}\right) \\
& -\left(\begin{array}{cc}
0 & 0 \\
-0.313 & 0.750
\end{array}\right)\left(\begin{array}{l}
\epsilon_{1, t-1} \\
\epsilon_{2, t-1}
\end{array}\right) .
\end{aligned}
$$

Table C.1 displays the relative frequency (in \%) of the orders selected by various standard and modified versions of the AIC criteria of a strong (Model I) candidates VARMA models, over the $N$ independent replications. Table C.1 shows that a standard AICc and a modified $\mathrm{AIC}_{M}$ have performed in the small samples sizes $(n=20$ and $n=50)$ and selected the true orders of the strong model. By contrast, when $n=20$ a standard AIC overfit the order $q$ and selected an $\operatorname{VARMA}(1,3)$, but did not perform well. In view of the observed relative frequency in Tables C.2 and C.3, the true orders $(1,1)$ (i.e. $\operatorname{VARMA}(1,1)$ model) are selected by all versions of the AIC criteria. They have similar performance, with a slight advantage to the standard versions.

Tables C.4 and C.5 display the relative frequency (in \%) of the orders selected by various standard and modified versions of the AIC criteria of weak candidates VARMA models, firstly with error term (7) (Model III) and secondly,

with error term (9) (Model IV). In view of the observed relative frequency, the 
true orders $(1,1)$ are selected by all versions of the $\mathbf{A I C}$ criteria. They have similar performance, with a slight advantage to the standard versions. 
Table C.1

Relative frequency (in \%) of the order selected by various standard and modified versions of the AIC criteria.

\begin{tabular}{|c|c|c|c|c|}
\hline \multirow{2}{*}{$\begin{array}{l}\text { Length } \\
n\end{array}$} & \multirow{2}{*}{$\begin{array}{l}\text { Order } \\
(p, q)\end{array}$} & \multicolumn{3}{|c|}{ Criteria Model I } \\
\hline & & $\mathrm{AIC}$ & $\mathrm{AICc}$ & $\mathrm{AIC}_{M}$ \\
\hline \multirow{9}{*}{20} & $(1,1)$ & 27.5 & 64.1 & 62.1 \\
\hline & $(2,2)$ & 0.2 & 0.0 & 0.5 \\
\hline & $(2,1)$ & 1.6 & 2.0 & 4.1 \\
\hline & $(1,2)$ & 19.3 & 22.2 & 14.9 \\
\hline & $(3,3)$ & 2.8 & 0.0 & 0.8 \\
\hline & $(3,2)$ & 3.5 & 0.0 & 1.3 \\
\hline & $(3,1)$ & 0.6 & 0.0 & 1.8 \\
\hline & $(2,3)$ & 11.0 & 0.1 & 2.1 \\
\hline & $(1,3)$ & 33.5 & 11.6 & 12.4 \\
\hline \multirow{9}{*}{50} & $(1,1)$ & 39.9 & 61.9 & 58.3 \\
\hline & $(2,2)$ & 0.1 & 0.0 & 0.3 \\
\hline & $(2,1)$ & 1.5 & 1.5 & 2.4 \\
\hline & $(1,2)$ & 11.8 & 12.0 & 9.6 \\
\hline & $(3,3)$ & 9.7 & 1.3 & 6.3 \\
\hline & $(3,2)$ & 3.0 & 2.0 & 3.2 \\
\hline & $(3,1)$ & 0.2 & 0.3 & 0.4 \\
\hline & $(2,3)$ & 24.5 & 13.6 & 15.5 \\
\hline & $(1,3)$ & 9.3 & 7.4 & 4.0 \\
\hline \multirow{9}{*}{100} & $(1,1)$ & 52.2 & 62.6 & 56.9 \\
\hline & $(2,2)$ & 0.1 & 0.1 & 0.1 \\
\hline & $(2,1)$ & 0.9 & 1.0 & 2.2 \\
\hline & $(1,2)$ & 6.3 & 6.7 & 6.9 \\
\hline & $(3,3)$ & 12.5 & 6.4 & 10.7 \\
\hline & $(3,2)$ & 1.7 & 1.3 & 2.2 \\
\hline & $(3,1)$ & 0.2 & 0.1 & 0.6 \\
\hline & $(2,3)$ & 22.5 & 18.7 & 18.0 \\
\hline & $(1,3)$ & 3.6 & 3.1 & 2.4 \\
\hline
\end{tabular}

I: Strong VARMA(1,1) model (C.1)-(6) 
Table C.2

Relative frequency (in \%) of the order selected by various standard and modified versions of the AIC criteria.

\begin{tabular}{|c|c|c|c|c|c|c|c|}
\hline \multirow{2}{*}{$\begin{array}{l}\text { Length } \\
n\end{array}$} & \multirow{2}{*}{$\begin{array}{l}\text { Order } \\
(p, q)\end{array}$} & \multicolumn{3}{|c|}{ Criteria Model I } & \multicolumn{3}{|c|}{ Criteria Model II } \\
\hline & & AIC & $\mathrm{AICc}$ & $\mathrm{AIC}_{M}$ & $\mathrm{AIC}$ & $\mathrm{AICc}$ & $\mathrm{AIC}_{M}$ \\
\hline \multirow{9}{*}{500} & $(1,1)$ & 82.0 & 83.4 & 74.4 & 62.6 & 63.7 & 59.2 \\
\hline & $(2,2)$ & 0.1 & 0.1 & 1.1 & 1.2 & 0.9 & 2.9 \\
\hline & $(2,1)$ & 0.8 & 0.8 & 2.1 & 1.6 & 1.5 & 3.9 \\
\hline & $(1,2)$ & 1.9 & 1.9 & 4.6 & 20.0 & 19.6 & 17.2 \\
\hline & $(3,3)$ & 11.4 & 10.3 & 12.9 & 9.2 & 9.2 & 10.8 \\
\hline & $(3,2)$ & 0.3 & 0.2 & 0.5 & 0.6 & 0.5 & 0.7 \\
\hline & $(3,1)$ & 0.0 & 0.0 & 0.3 & 0.0 & 0.0 & 0.7 \\
\hline & $(2,3)$ & 2.1 & 1.9 & 2.4 & 1.7 & 1.7 & 2.1 \\
\hline & $(1,3)$ & 1.4 & 1.4 & 1.7 & 3.1 & 2.9 & 2.5 \\
\hline \multirow{9}{*}{2000} & $(1,1)$ & 90.6 & 91.1 & 80.2 & 79.1 & 79.3 & 73.5 \\
\hline & $(2,2)$ & 0.8 & 0.7 & 1.7 & 0.3 & 0.3 & 1.5 \\
\hline & $(2,1)$ & 0.2 & 0.2 & 1.6 & 1.9 & 1.9 & 4.8 \\
\hline & $(1,2)$ & 1.7 & 1.5 & 4.2 & 11.9 & 11.8 & 10.4 \\
\hline & $(3,3)$ & 5.5 & 5.3 & 10.5 & 4.6 & 4.5 & 4.9 \\
\hline & $(3,2)$ & 0.2 & 0.2 & 0.1 & 0.2 & 0.2 & 0.4 \\
\hline & $(3,1)$ & 0.0 & 0.0 & 0.1 & 0.0 & 0.0 & 0.3 \\
\hline & $(2,3)$ & 0.4 & 0.4 & 0.1 & 0.6 & 0.6 & 1.4 \\
\hline & $(1,3)$ & 0.6 & 0.6 & 1.5 & 1.4 & 1.4 & 2.8 \\
\hline
\end{tabular}

I: Strong VARMA(1,1) model (C.1)-(6)

II: Weak VARMA(1,1) model (C.1)-(8) 
Table C.3

Relative frequency (in \%) of the order selected by various standard and modified versions of the AIC criteria.

\begin{tabular}{|c|c|c|c|c|c|c|c|}
\hline \multirow{2}{*}{$\begin{array}{l}\text { Length } \\
n\end{array}$} & \multirow{2}{*}{$\begin{array}{l}\text { Order } \\
(p, q)\end{array}$} & \multicolumn{3}{|c|}{ Criteria Model I } & \multicolumn{3}{|c|}{ Criteria Model II } \\
\hline & & AIC & $\mathrm{AICc}$ & $\mathrm{AIC}_{M}$ & $\mathrm{AIC}$ & $\mathrm{AICc}$ & $\mathrm{AIC}_{M}$ \\
\hline \multirow{9}{*}{5,000} & $(1,1)$ & 82.0 & 83.4 & 74.4 & 81.0 & 81.0 & 74.0 \\
\hline & $(2,2)$ & 0.1 & 0.1 & 1.1 & 0.0 & 0.0 & 0.0 \\
\hline & $(2,1)$ & 0.8 & 0.8 & 2.1 & 3.0 & 3.0 & 6.0 \\
\hline & $(1,2)$ & 1.9 & 1.9 & 4.6 & 11.0 & 11.0 & 7.0 \\
\hline & $(3,3)$ & 11.4 & 10.3 & 12.9 & 4.0 & 4.0 & 6.0 \\
\hline & $(3,2)$ & 0.3 & 0.2 & 0.5 & 0.0 & 0.0 & 2.0 \\
\hline & $(3,1)$ & 0.0 & 0.0 & 0.3 & 0.0 & 0.0 & 2.0 \\
\hline & $(2,3)$ & 2.1 & 1.9 & 2.4 & 0.0 & 0.0 & 1.0 \\
\hline & $(1,3)$ & 1.4 & 1.4 & 1.7 & 1.0 & 1.0 & 2.0 \\
\hline \multirow{9}{*}{10,000} & $(1,1)$ & 90.6 & 91.1 & 80.2 & 75.0 & 75.0 & 70.0 \\
\hline & $(2,2)$ & 0.8 & 0.7 & 1.7 & 0.0 & 0.0 & 3.0 \\
\hline & $(2,1)$ & 0.2 & 0.2 & 1.6 & 0.0 & 0.0 & 5.0 \\
\hline & $(1,2)$ & 1.7 & 1.5 & 4.2 & 20.0 & 20.0 & 11.0 \\
\hline & $(3,3)$ & 5.5 & 5.3 & 10.5 & 1.0 & 1.0 & 4.0 \\
\hline & $(3,2)$ & 0.2 & 0.2 & 0.1 & 0.0 & 0.0 & 1.0 \\
\hline & $(3,1)$ & 0.0 & 0.0 & 0.1 & 0.0 & 0.0 & 3.0 \\
\hline & $(2,3)$ & 0.4 & 0.4 & 0.1 & 1.0 & 1.0 & 1.0 \\
\hline & $(1,3)$ & 0.6 & 0.6 & 1.5 & 3.0 & 3.0 & 2.0 \\
\hline
\end{tabular}

I: Strong VARMA(1,1) model (C.1)-(6)

II: Weak VARMA(1,1) model (C.1)-(8) 
Table C.4

Relative frequency (in \%) of the order selected by various standard and modified versions of the AIC criteria.

\begin{tabular}{|c|c|c|c|c|c|c|c|}
\hline \multirow{2}{*}{$\begin{array}{l}\text { Length } \\
n\end{array}$} & \multirow{2}{*}{$\begin{array}{l}\text { Order } \\
(p, q)\end{array}$} & \multicolumn{3}{|c|}{ Criteria Model III } & \multicolumn{3}{|c|}{ Criteria Model IV } \\
\hline & & AIC & $\mathrm{AICc}$ & $\mathrm{AIC}_{M}$ & $\mathrm{AIC}$ & $\mathrm{AICc}$ & $\mathrm{AIC}_{M}$ \\
\hline \multirow{9}{*}{500} & $(1,1)$ & 74.3 & 75.6 & 67.9 & 82.1 & 83.0 & 75.0 \\
\hline & $(2,2)$ & 0.3 & 0.3 & 1.0 & 0.3 & 0.3 & 1.0 \\
\hline & $(2,1)$ & 0.8 & 0.8 & 2.9 & 0.3 & 0.2 & 1.4 \\
\hline & $(1,2)$ & 8.4 & 8.2 & 11.4 & 1.2 & 1.1 & 4.3 \\
\hline & $(3,3)$ & 8.5 & 8.0 & 7.0 & 12.5 & 11.8 & 13.2 \\
\hline & $(3,2)$ & 0.4 & 0.4 & 0.7 & 0.1 & 0.1 & 0.6 \\
\hline & $(3,1)$ & 0.0 & 0.0 & 0.4 & 0.0 & 0.0 & 0.1 \\
\hline & $(2,3)$ & 6.1 & 5.9 & 6.0 & 2.3 & 2.3 & 1.8 \\
\hline & $(1,3)$ & 1.2 & 0.8 & 2.7 & 1.2 & 1.2 & 2.6 \\
\hline \multirow{9}{*}{2000} & $(1,1)$ & 84.0 & 84.2 & 73.4 & 90.9 & 90.9 & 87.3 \\
\hline & $(2,2)$ & 0.3 & 0.3 & 1.8 & 0.0 & 0.0 & 0.9 \\
\hline & $(2,1)$ & 0.8 & 0.8 & 3.4 & 0.3 & 0.3 & 0.9 \\
\hline & $(1,2)$ & 7.8 & 7.8 & 8.4 & 1.8 & 1.8 & 3.2 \\
\hline & $(3,3)$ & 3.2 & 3.2 & 7.2 & 6.7 & 6.7 & 5.8 \\
\hline & $(3,2)$ & 0.1 & 0.1 & 0.4 & 0.1 & 0.1 & 0.1 \\
\hline & $(3,1)$ & 0.0 & 0.0 & 0.7 & 0.0 & 0.0 & 0.0 \\
\hline & $(2,3)$ & 0.5 & 0.5 & 0.5 & 0.0 & 0.0 & 0.5 \\
\hline & $(1,3)$ & 3.3 & 3.1 & 4.2 & 0.2 & 0.2 & 1.3 \\
\hline
\end{tabular}

III: Weak VARMA $(1,1)$ model GARCH (C.1)-(7)

IV: Weak VARMA(1,1) model (C.1)-(9) 
Table C.5

Relative frequency (in \%) of the order selected by various standard and modified versions of the AIC criteria.

\begin{tabular}{|c|c|c|c|c|c|c|c|}
\hline \multirow{2}{*}{$\begin{array}{l}\text { Length } \\
n\end{array}$} & \multirow{2}{*}{$\begin{array}{l}\text { Order } \\
(p, q)\end{array}$} & \multicolumn{3}{|c|}{ Criteria Model III } & \multicolumn{3}{|c|}{ Criteria Model IV } \\
\hline & & AIC & $\mathrm{AICc}$ & $\mathrm{AIC}_{M}$ & $\mathrm{AIC}$ & AICc & $\mathrm{AIC}_{M}$ \\
\hline \multirow{9}{*}{5,000} & $(1,1)$ & 82.0 & 82.0 & 79.0 & 95.0 & 95.0 & 85.0 \\
\hline & $(2,2)$ & 0.0 & 0.0 & 0.0 & 0.0 & 0.0 & 2.0 \\
\hline & $(2,1)$ & 0.0 & 0.0 & 1.0 & 0.0 & 0.0 & 1.0 \\
\hline & $(1,2)$ & 8.0 & 8.0 & 9.0 & 1.0 & 1.0 & 2.0 \\
\hline & $(3,3)$ & 3.0 & 3.0 & 4.0 & 2.0 & 2.0 & 6.0 \\
\hline & $(3,2)$ & 0.0 & 0.0 & 1.0 & 0.0 & 0.0 & 0.0 \\
\hline & $(3,1)$ & 0.0 & 0.0 & 0.0 & 0.0 & 0.0 & 1.0 \\
\hline & $(2,3)$ & 0.0 & 0.0 & 1.0 & 0.0 & 0.0 & 0.0 \\
\hline & $(1,3)$ & 7.0 & 7.0 & 5.0 & 2.0 & 2.0 & 3.0 \\
\hline \multirow{9}{*}{10,000} & $(1,1)$ & 89.0 & 89.0 & 84.0 & 96.0 & 96.0 & 87.0 \\
\hline & $(2,2)$ & 1.0 & 1.0 & 3.0 & 0.0 & 0.0 & 0.0 \\
\hline & $(2,1)$ & 0.0 & 0.0 & 1.0 & 0.0 & 0.0 & 1.0 \\
\hline & $(1,2)$ & 8.0 & 8.0 & 8.0 & 3.0 & 3.0 & 7.0 \\
\hline & $(3,3)$ & 0.0 & 0.0 & 0.0 & 1.0 & 1.0 & 5.0 \\
\hline & $(3,2)$ & 0.0 & 0.0 & 0.0 & 0.0 & 0.0 & 0.0 \\
\hline & $(3,1)$ & 0.0 & 0.0 & 0.0 & 0.0 & 0.0 & 0.0 \\
\hline & $(2,3)$ & 0.0 & 0.0 & 1.0 & 0.0 & 0.0 & 0.0 \\
\hline & $(1,3)$ & 2.0 & 2.0 & 3.0 & 0.0 & 0.0 & 0.0 \\
\hline
\end{tabular}

III: Weak VARMA(1,1) model GARCH (C.1)-(7)

IV: Weak VARMA(1,1) model (C.1)-(9) 
Table C.6

Modified version of asymptotic probabilities of overfitting by $\ell=d^{2}\left(\ell_{1}+\ell_{2}\right)$ parameters for bivariate VARMA models of various versions of AIC criteria.

\begin{tabular}{|c|c|c|c|c|c|c|c|}
\hline \multirow{2}{*}{$\begin{array}{l}\text { Length } \\
n\end{array}$} & \multirow{2}{*}{$\begin{array}{l}\text { Order } \\
\left(\ell_{1}, \ell_{2}\right)\end{array}$} & \multicolumn{3}{|c|}{$\mathbf{P}_{W}$ Model I } & \multicolumn{3}{|c|}{$\mathbf{P}_{W}$ Model II } \\
\hline & & $\mathbf{P}_{W}^{A I C}$ & $\mathbf{P}_{W}^{A I C c}$ & $\mathbf{P}_{W}^{A I C_{M}}$ & $\mathbf{P}_{W}^{A I C}$ & $\mathbf{P}_{W}^{A I C c}$ & $\mathbf{P}_{W}^{A I C_{M}}$ \\
\hline \multirow{8}{*}{500} & $(1,0)$ & 0.009 & 0.009 & 0.028 & 0.057 & 0.056 & 0.104 \\
\hline & $(0,1)$ & 0.025 & 0.024 & 0.060 & 0.237 & 0.231 & 0.228 \\
\hline & $(1,1)$ & 0.003 & 0.002 & 0.021 & 0.086 & 0.079 & 0.136 \\
\hline & $(0,2)$ & 0.016 & 0.016 & 0.036 & 0.130 & 0.117 & 0.139 \\
\hline & $(2,0)$ & 0.002 & 0.002 & 0.011 & 0.016 & 0.016 & 0.057 \\
\hline & $(1,2)$ & 0.027 & 0.024 & 0.036 & 0.073 & 0.065 & 0.103 \\
\hline & $(2,1)$ & 0.006 & 0.005 & 0.011 & 0.043 & 0.039 & 0.076 \\
\hline & $(2,2)$ & 0.126 & 0.114 & 0.140 & 0.127 & 0.121 & 0.168 \\
\hline \multirow{8}{*}{2000} & $(1,0)$ & 0.007 & 0.007 & 0.026 & 0.083 & 0.081 & 0.124 \\
\hline & $(0,1)$ & 0.020 & 0.017 & 0.058 & 0.258 & 0.251 & 0.221 \\
\hline & $(1,1)$ & 0.009 & 0.009 & 0.022 & 0.103 & 0.102 & 0.133 \\
\hline & $(0,2)$ & 0.010 & 0.010 & 0.036 & 0.147 & 0.146 & 0.141 \\
\hline & $(2,0)$ & 0.001 & 0.001 & 0.006 & 0.026 & 0.025 & 0.069 \\
\hline & $(1,2)$ & 0.005 & 0.005 & 0.011 & 0.071 & 0.071 & 0.096 \\
\hline & $(2,1)$ & 0.005 & 0.005 & 0.011 & 0.053 & 0.052 & 0.082 \\
\hline & $(2,2)$ & 0.057 & 0.055 & 0.108 & 0.073 & 0.071 & 0.122 \\
\hline
\end{tabular}

I: Strong VARMA(1,1) model (C.1)-(6)

II: Weak VARMA(1,1) model (C.1)-(8) 
Table C.6 displays the modified version of asymptotic probabilities of overfitting by $\ell=d^{2}\left(\ell_{1}+\ell_{2}\right)$ parameters for bivariate VARMA models of various versions of AIC criteria. Table C.6 shows clearly that the $\mathrm{AIC}_{M}$ criterion is not consistent in the weak and strong VARMA cases, since his probability of overfitting is not zero. The modified asymptotic probabilities of overfitting of the standard and modified versions of the AIC criteria are similar in the two cases. Note that the asymptotic probabilities of overfitting of the $\mathrm{AIC}_{M}$ criterion decreases when $n$ is large.

\section{Others simulations on strong and weak vector moving average (VMA) case}

We simulated $N$ independent trajectories of different sizes of bivariate VMA(1) model with the strong Gaussian and the weak noise above-mentioned. We took $N=1,000$ when the sample size $n \leq 2000$ and $N=1,00$ in the opposite case. For each of these $N$ replications of VMA(1) model, we will fit 6 candidates models (i.e. $\operatorname{VMA}(k)$ models with $k=1, \ldots, 6)$. The QML method was used to fit candidates bivariate VMA models of order $1, \ldots, 6$; standard and modified versions of AIC criteria were used to select among the candidates models.

To generate the strong and weak VMA(1) models, we consider the bivariate model of the form

$$
\left(\begin{array}{l}
X_{1, t} \\
X_{2, t}
\end{array}\right)=\left(\begin{array}{c}
\epsilon_{1, t} \\
\epsilon_{2, t}
\end{array}\right)-\left(\begin{array}{ll}
0.5 & 0.1 \\
0.4 & 0.5
\end{array}\right)\left(\begin{array}{l}
\epsilon_{1, t-1} \\
\epsilon_{2, t-1}
\end{array}\right), \quad \Sigma_{0}=\left[\begin{array}{ll}
1 & 0 \\
0 & 1
\end{array}\right]
$$

Table D.1 displays the relative frequency (in \%) of the order selected by various standard and modified versions of the AIC criteria of a strong (Model I) $\operatorname{VMA}(k)$ candidates models, for $k=1, \ldots, 6$, over the $N$ independent replications. Table D.1 shows that the standard AIC criteria have overfit the order $q$ in the small sample size (i.e. $n=50$ ) and selected a $\operatorname{VMA}(6)$ model. By contrast, the modified criterion selected a VMA(1) model. In view of the observed relative frequency, when $n>50$, the order $q=1$ (i.e. $\operatorname{VMA}(1)$ model) is selected by all versions of the AIC criteria, but the modified criterion has clearly hight performance.

Table D.2 displays the relative frequency (in \%) of the order selected by various standard and modified versions of the AIC criteria of a strong (Model I) and weak (Model II, with error term (8)) $\operatorname{VMA}(k)$ candidates models, for $k=1, \ldots, 6$, over the $N$ independent replications. Table D.2 shows that the standard AIC criteria have overfit the order $q$ in the small sample size $(n=20$ 
and $n=50$ ). In view of the observed relative frequency, the order $q=1$ (i.e. VMA(1) model) is selected by all versions of the AIC criteria in Models I and II. As expected in Model II, the observed relative frequency of the standard AIC criteria is very smaller than a modified one. Table D.2 shows also that the standard AIC criteria clearly did not perform well here, and they have tendency to overestimate the order $q=3$. By contrast, in Model I all versions of the AIC criteria have the same performance.

Table D.3 displays the relative frequency (in \%) of the order selected by various standard and modified versions of the AIC criteria of a weak $\operatorname{VMA}(k)$ candidates models for $k=1, \ldots, 6$, firstly with error term (7) (Model III) and secondly with error term (9) (Model IV). In view of the observed relative frequency, a VMA(1) model is selected by all versions of the AIC criteria and they have the same performance in Model IV. By contrast, Table D.3 shows that a modified criterion has clearly hight performance in Model III. 
Table D.1

Relative frequency (in \%) of the order selected by various standard and modified versions of the AIC criteria.

\begin{tabular}{|c|c|c|c|c|}
\hline Length & Order & \multicolumn{3}{|c|}{ Criteria Model I } \\
\hline$n$ & $q$ & AIC & $\mathrm{AICc}$ & $\mathrm{AIC}_{M}$ \\
\hline & 1 & 1.8 & 5.8 & 53.4 \\
\hline & 2 & 0.0 & 0.0 & 1.3 \\
\hline & 3 & 1.8 & 4.5 & 9.7 \\
\hline \multirow[t]{6}{*}{50} & 4 & 7.4 & 12.0 & 9.4 \\
\hline & 5 & 25.0 & 28.8 & 13.5 \\
\hline & 6 & 64.0 & 48.9 & 12.7 \\
\hline & 1 & 65.3 & 72.5 & 85.3 \\
\hline & 2 & 0.2 & 0.1 & 0.5 \\
\hline & 3 & 6.5 & 5.1 & 5.2 \\
\hline \multirow[t]{6}{*}{100} & 4 & 3.2 & 2.4 & 1.4 \\
\hline & 5 & 5.9 & 4.8 & 2.9 \\
\hline & 6 & 18.9 & 15.1 & 4.7 \\
\hline & 1 & 92.4 & 93.8 & 94.2 \\
\hline & 2 & 0.0 & 0.0 & 0.0 \\
\hline & 3 & 3.1 & 2.7 & 3.2 \\
\hline \multirow[t]{3}{*}{200} & 4 & 1.8 & 1.9 & 1.2 \\
\hline & 5 & 1.4 & 1.0 & 0.8 \\
\hline & 6 & 1.3 & 0.6 & 0.6 \\
\hline
\end{tabular}

I: Strong VMA(1) model (D.1)-(6) 
Table D.2

Relative frequency (in \%) of the order selected by various standard and modified versions of the AIC criteria.

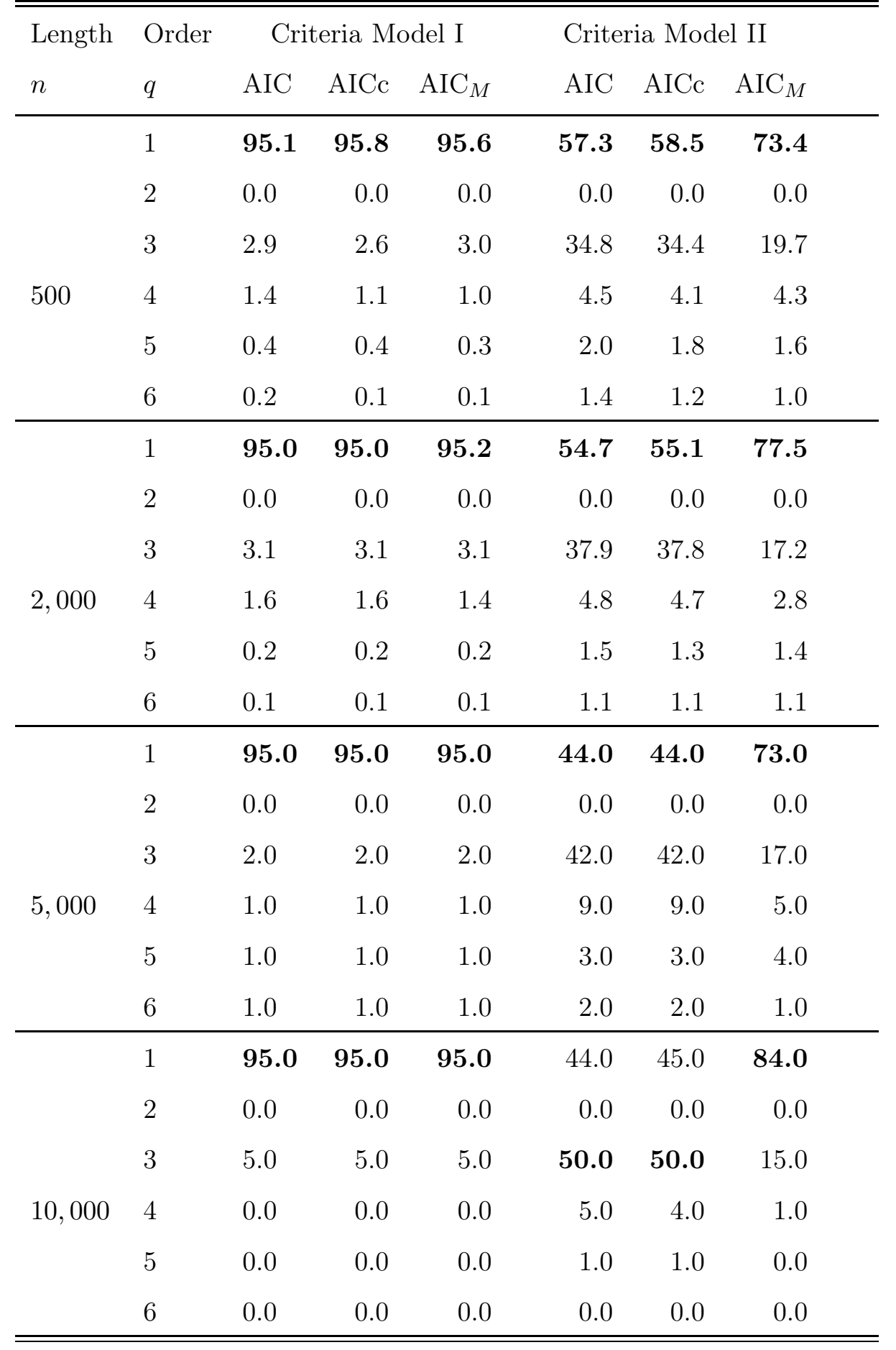

I: Strong VMA(1) model (D.1)-(6); II: Weak VMA(1) model (D.1)-(8) 
Table D.3

Relative frequency (in \%) of the order selected by various standard and modified versions of the criteria AIC.

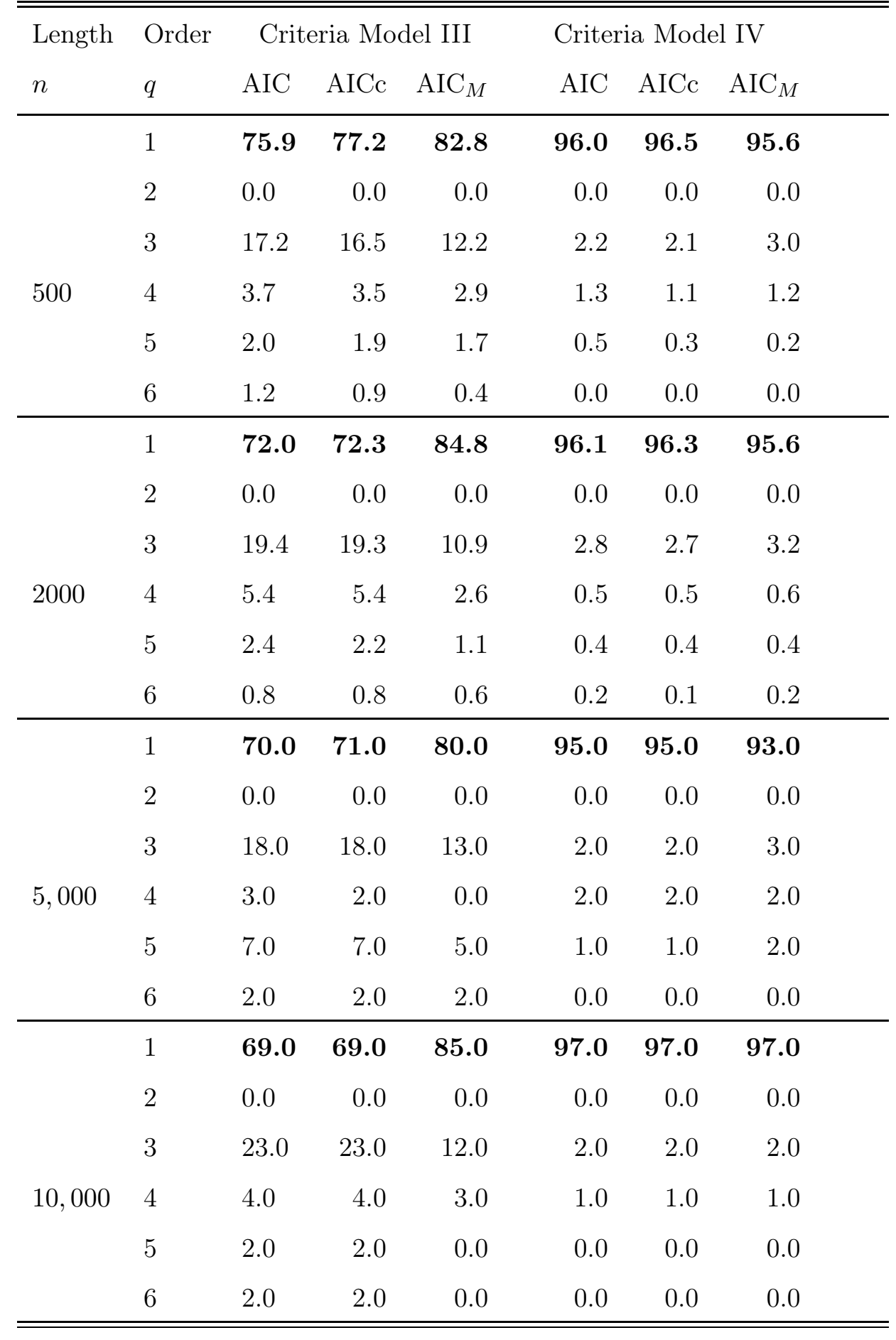

III: Weak VMA(1) model (D.1)-(7), IV: Weak VMA(1) model (D.1)-(9) 